VOL. 23: Supplement 1

I

JANUARY-APRIL 2018

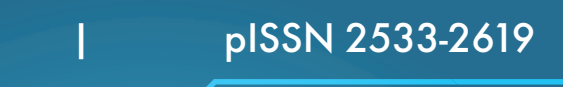

\section{RIVISTA DI}

PSICOPATOLOGLA FORENSE

MEDICINA LEGA

CRIMINO

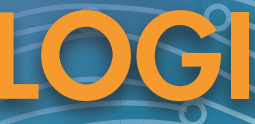

YA

orte a cara

Periodico quadrimestrale a caratiere scientifiec

UNIVERSITÀ DEGLI STUDIDIROMA" "SAPIENZA

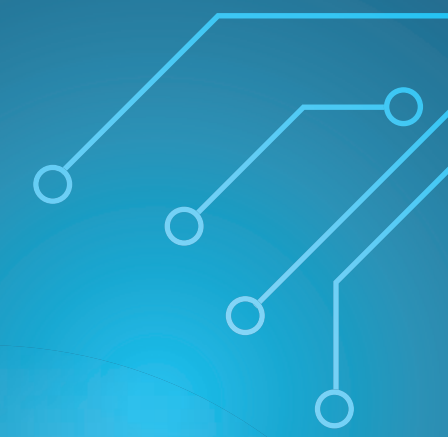




\section{Rivista di Psicopatologia Forense, Medicina Legale, Criminologia}

ISSN 2533-2619

DiretToRe ResponsabiLe

Natale Mario di Luca

Vice DiretTore ResponsabiLe

Enrico Marinelli

COORDINATORE RESPONSABILE E

DIREZIONE SCIENTIFICA DELLA RIVISTA

Vincenzo Mastronardi

Responsabile del Settore Medico Legale

Simona Zaami

RESPONSABILE DI REDAZIONE

Monica Calderaro

Responsabili Scientifici del Settore Giuridico

Luca Montanari Vergallo

Giovanni Neri

Giovanni Cicchitelli

RESPONSABILE PER I RAPPORTI ITALIA-AMERICA LATINA Lucrecia Emilce Vega Gramunt

Gli elaborati vanno inviati al Prof. Vincenzo Mastronardi al seguente indirizzo E-mail:

vincenzo.mastronardi@uniromal.it

Comitato Scientifico

M. Calderaro

G. Cicchitelli

G. Maestoso

E. Marinelli

V. Mastronardi

G. Montanari Vergallo

G. Neri

L. Pacetti

E. Pizzi

P. Ricci

M. Senesi

S. Zaami

\section{Comitato Consultivo}

J. Aubut, Montreal, Canada

J. Arboleda-Florez, Queens, USA

R. Bisi, University of Bologna, Italy

G. Bisogni, Unint, Roma, Italy

J.A. Bujan, Buenos Aires, Argentina

M. Calderaro, IISCPF, Roma, Italy

C. Ciallella, Sapienza, Roma, Italy

N.M. di Luca, Sapienza, Roma, Italy

U. Fornari, Torino, Italy

T. Gutheil, Harvard, USA

A.M. Lapenta, Torino, Italy

E. Marinelli, Roma, Italy

V. Mastronardi, IISCPF, Roma, Italy

I. Merzagora, Università degli Studi, Milano, Italy

G. Montanari Vergallo, Sapienza, Roma, Italy

G. Neri, UPM, Milano, Italy

A. Slaby, New York, USA

G. Palermo †, Las Vegas, USA

S. Ricci, Sapienza, Roma, Italy

S.P. Rigonatti, Sao Paulo, Brazil

J.A. Sereni, Maimonides, Buenos Aires, Argentina

L. Tancredi, New York, USA

L.E. Vega Gramunt, IISCPF, Buenos Aires, Argentina

D.N. Weisstub, Montreal, Canada

G. Woods, Berkley, USA

S. Zaami, Sapienza, Roma, Italy

\section{EDItorial STAFF}

Nadia Moscato, Managing Editor

Claudia Castellano, Production Editor

Tiziano Taccini, Technical Support

\section{Rivista di Psicopatologia Forense, Medicina Legale, Criminologia}

Periodico quadrimestrale a carattere scientifico di proprietà della Università degli Studi di Roma "Sapienza".

\section{SAPIENZA}

UNIVERSITÀ DI ROMA

Dipartimento di Scienze Anatomiche, Istologiche, Medico Legali e dell'Apparato Locomotore. Sezione di Medicina Legale.

Università degli Studi di Roma "Sapienza".

Tutti i diritti sono riservati: nessuna parte di questa pubblicazione può essere riprodotta, trasmessa e memorizzata in qualsiasi forma e con qualsiasi mezzo. Per quanto non espressamente richiamato valgono le norme delle Leggi sulla Stampa e le norme internazionali sul Copyright.

Registrazione al Tribunale Civile di Roma $n^{\circ} 00325 / 96(28.06 .1996)$.

Stampato: aprile 2018

ONLINE PUBLICATION AND PRINTING BY PAGEPress Publications via A. Cavagna Sangiuliani, 5 27100 Pavia, Italy

Tel: +39.0382.464340 - Fax: +39.0382.34872 info@pagepress.org - www.pagepress.org 


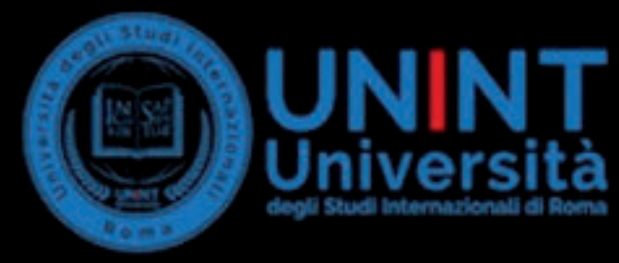

$$
\text { Pipla }
$$

Accademia It. di Criminologia

Sistema Penale

Investigazioni e Sicurezza

PRIMO CONGRESSO NAZIONALE DELLA

ACCADEMIA ITALIANA DI CRIMINOLOGIA,

SISTEMA PENALE, INVESTIGAZIONI E

SICUREZZA
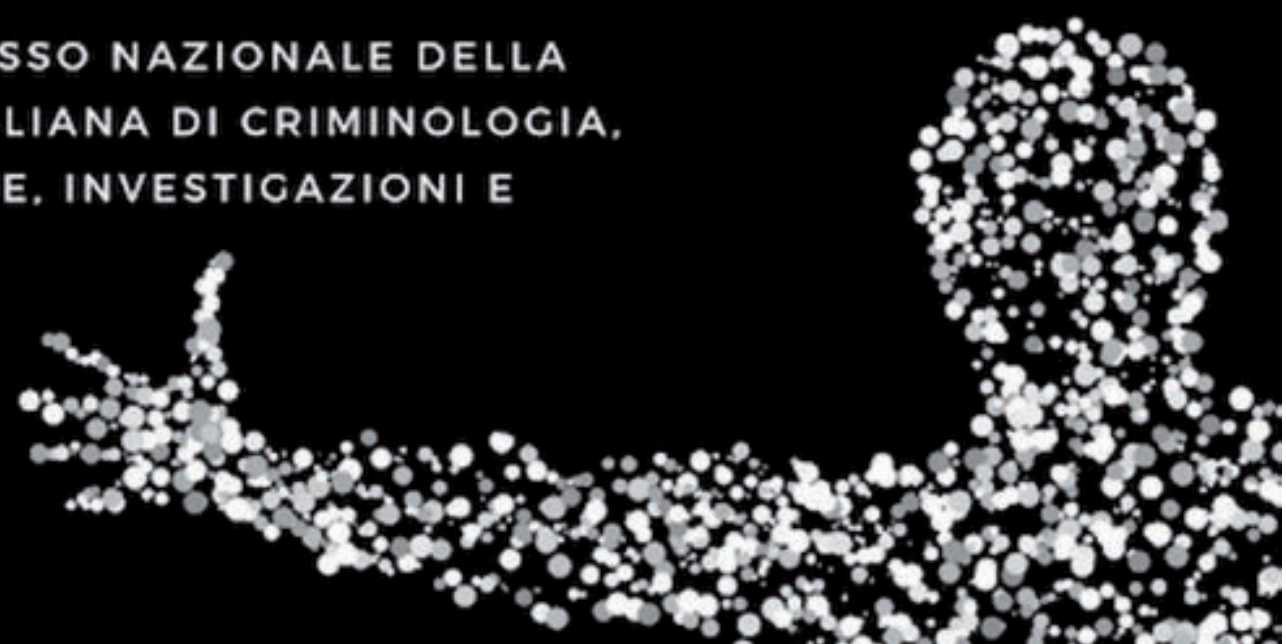

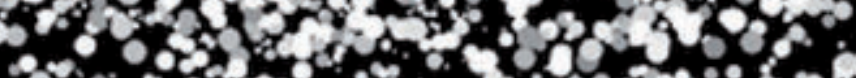

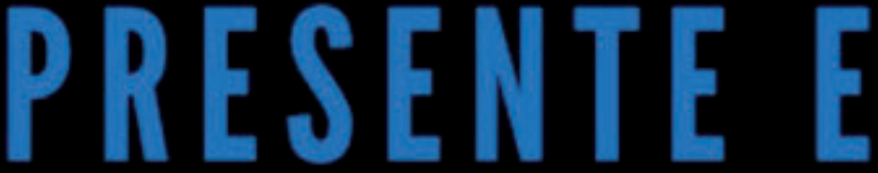

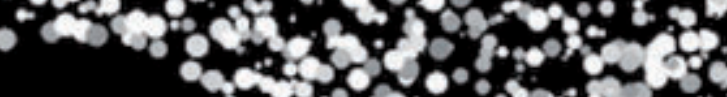
ats.8.

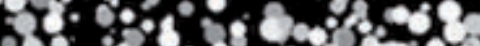

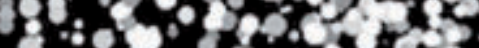

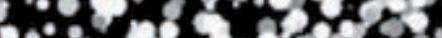

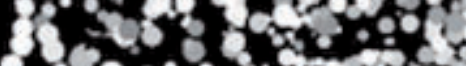
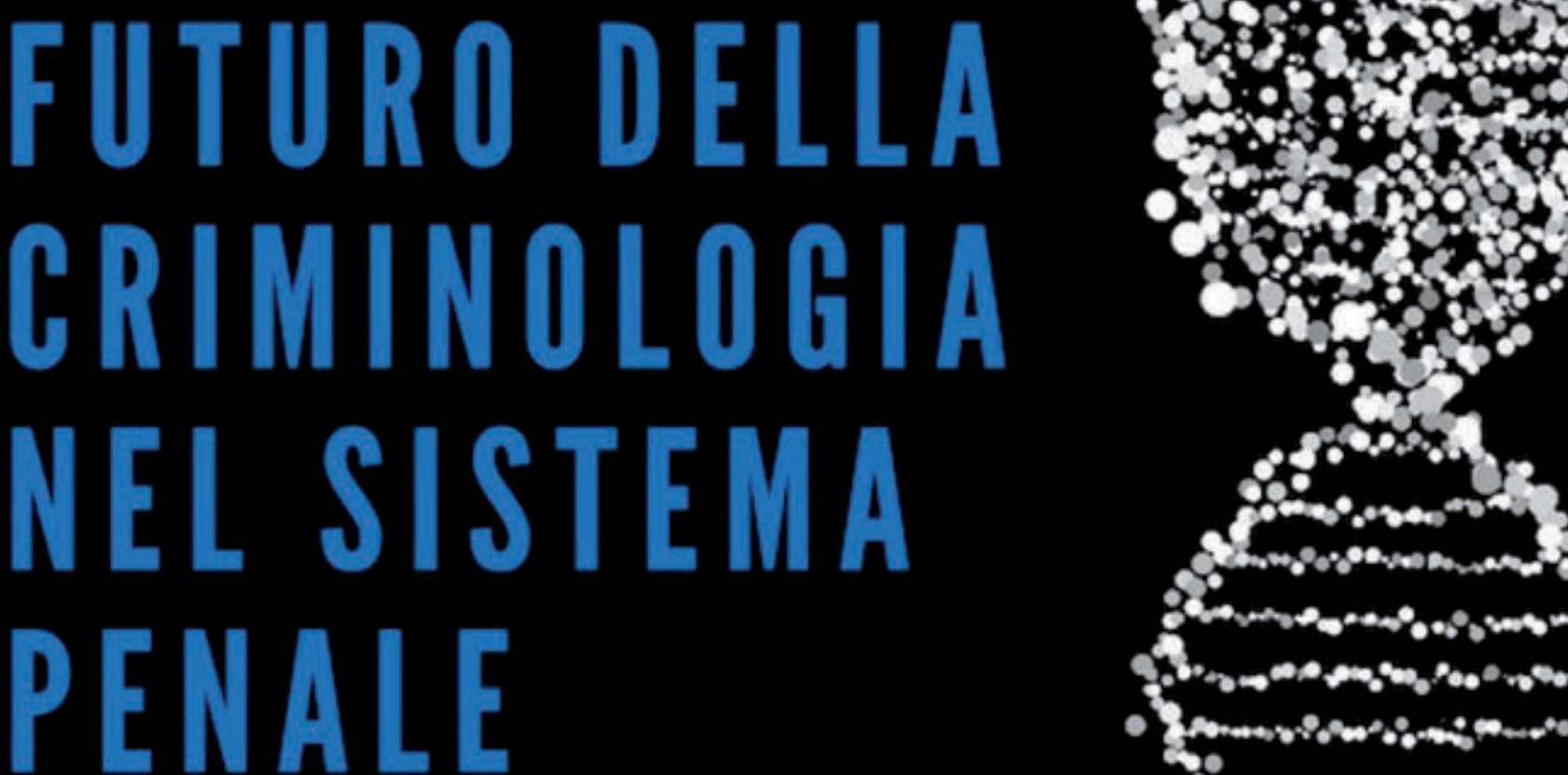

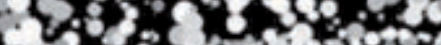

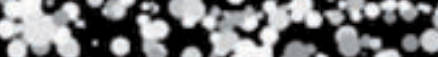

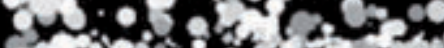
no.

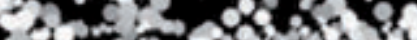

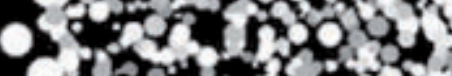

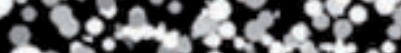
$C$ S. $2<$. sal $02000 \%$

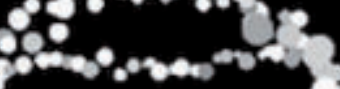
8

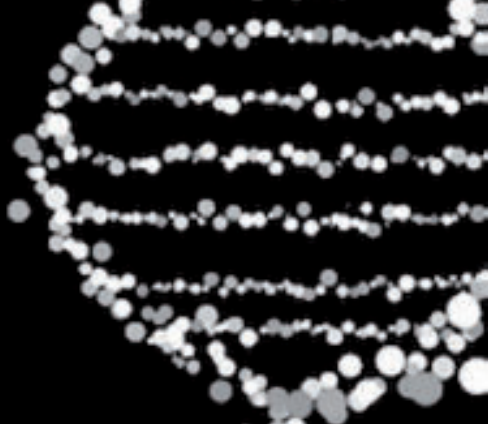

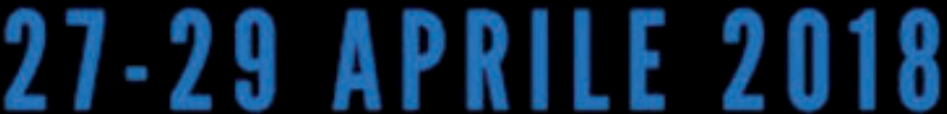

UNIVERSITÀ DEGLI STUDI INTERNAZIONALI DI ROMA - UNINT VIA CRISTOFORO COLOMBO, 200 - 00147, ROMA

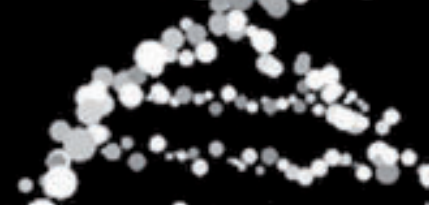




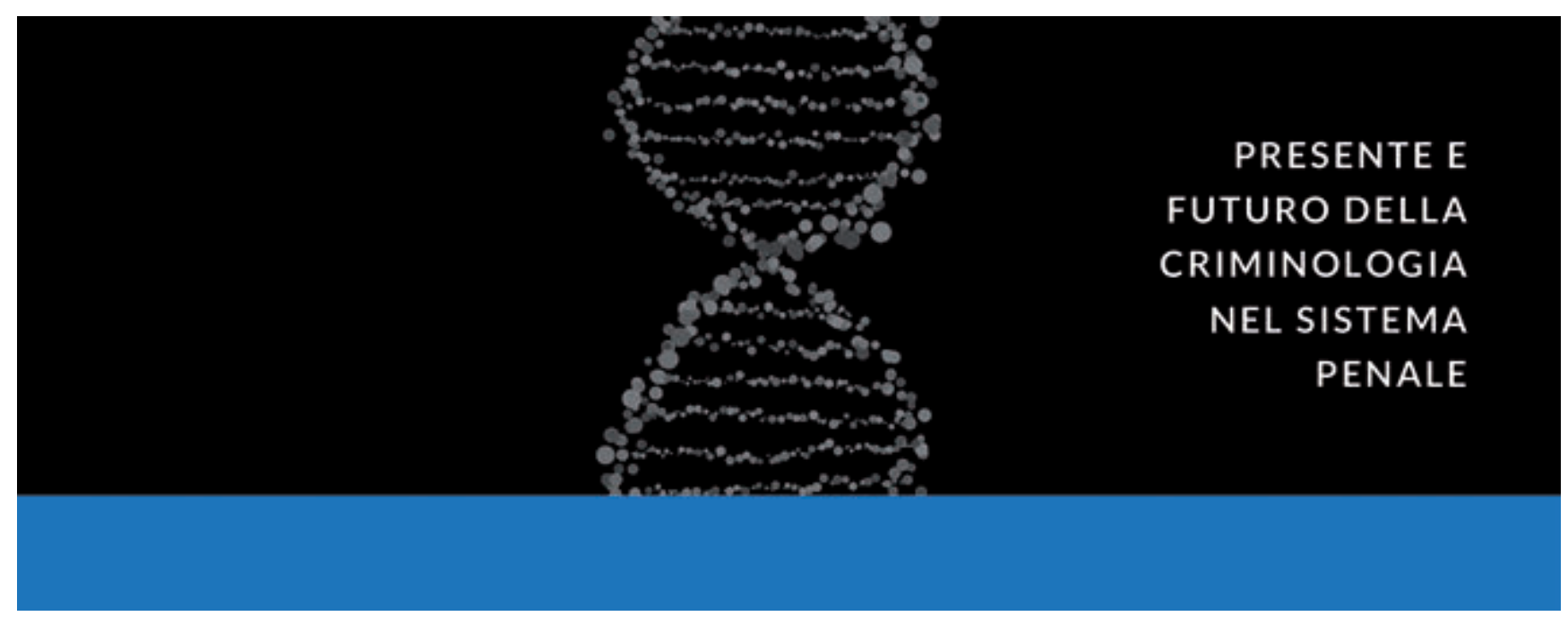

\title{
PRESENTE E FUTURO DELLA CRIMINOLOGIA NEL SISTEMA PENALE
}

\author{
I Congresso dell'Accademia Italiana di Criminologia, \\ Sistema penale, Investigazioni e Sicurezza
}

Roma, 27-29 aprile 2018

Università degli Studi Internazionali di Roma - UNINT

\section{ORGANIZZATORI}

\author{
Enrico Marinelli \\ Sezione di Medicina Legale Sapienza Università di Roma
}

Vincenzo Mastronardi

Presidente dell'Accademia Italiana di Criminologia, Sistema Penale, Investigazione e Sicurezza

Lello Spoletini

Direttore Associazione Tutela dei Diritti

Simona Zaami

Sezione di Medicina Legale Sapienza Università di Roma

Matteo Santini

Consigliere dell'Ordine degli Avvocati di Roma

Giovanni Neri

Segretario dell'Accademia Italiana di Criminologia, Sistema Penale, Investigazione e Sicurezza

Gianluca Montanari Vergallo

Sezione di Medicina Legale Sapienza Università di Roma

Monica Calderaro

Direttore dell'Istituto Internazionale di Scienze Criminologiche e Psicopatologico-Forensi

Lucrecia Vega Gramunt

Responsabile per la Spagna e l'America Latina dell'Istituto Internazionale di Scienze Criminologiche e Psicopatologico-Forensi 


\section{APRILE 2018}

14:30 - 17:00 PRIMA SESSIONE PLENARIA

Aula Magna

Ciro Sbailò, Università degli Studi Internazionali di Roma - UNINT, Italy

RELAZIONI INTRODUTTIVE

Javier Alejandro Bujan, Rettor del'Istituto Universitario Nazional de Derecios Humanos "Madres de Plaza de Mayo", Buenos Aires, Argentina

Cesare Mirabelli, Presidente emerito della Corte Costituzionale, ex vicepresidente del Csm, Professore emerito Università degli Studi di Roma "Tor Vergata", Italy

Natale Mario di Luca, Responsabile Sezione dipartimentale di Medicina Legale, Sapienza Università di Roma, Italy

Jorge A. Sereni, Presidente della Fondazione Civitas et Lex, Buenos Aires, Argentina

\section{TECNICHE DI NEGOZIAZIONE E PROFILING}

PRESENTA LA SESSIONE

Vincenzo Mastronardi, già titolare della Cattedra di Psicopatologia forense, Sapienza Università di Roma, Italy

Gregory Vecchi, Profiler già FBI supervisor e docente agenti speciali FBI; Esperto dell'FBI in tecniche di Negoziazione e profiling Dipartimento di giustizia penale, studi giuridici e lavoro sociale, Missouri Western State University, USA

Il Modello della Negoziazione risolutiva: le tecniche di negoziazione in caso di presa di ostaggi

Break

$17: 15-18: 15$

$18: 15-19: 15$

$17: 15-18: 15$

\section{WORKSHOP N. 1}

Vittimologia

PRESIDENTI

Antonello Crisci, Massimiliano Fioravanti

1. La vittimizzazione delle prostitute nigeriane tramite la ritualità JuJu: meccanismi psicologici e basi antropologiche Giuseppe Gino Saladini

2. I vissuti emotivi della vittima durante e dopo l'abuso sessuale infantile Daniela Labattaglia

3. Stalking nei confronti di operatori di contesti socio-sanitari: risultati preliminari di una ricerca nella regione Sardegna Francesca Manunza, Vincenzo Mastronardi, Monica Calderaro

4. L'istigazione al suicidio e modalità di estrinsecazione Francesca Arpino

\section{WORKSHOP N. 2}

Coppie criminali

PRESIDENTE

Lello Spoletini

1. Storie maledette. Barbie e Ken, la coppia assassina che ha sconvolto l'Ontario: disturbo psicotico condiviso? Monica Calderaro

2. La coppia dell'acido: un patto perverso tra immaturità e dipendenza. Uno studio grafologico Elena Manetti

3. Le coppie criminali. Dalla "folie à deux" al vizio di mente: una disamina di delitti efferati perpetrati in coppia Francesca Righi

\section{WORKSHOP N. 3}

I profili delle vittime di famiglia e le dipendenze

Aula 8

PRESIDENTI

Paolo Capri, Pietro Mazzei

1. Rivediamo il Concetto di Vittimologia Giovanni Neri

2. Dipendenza affettiva. Quando un sentimento diventa una droga Danila Pescina

3. Prevenire e ridurre le dipendenze nella pre-adolescenza: il Coping Power Program Gennarina Pirri

4. Uomini che odiano le donne. Donne che odiano gli uomini. Quando la fine di un amore può rovinare la vita Danila Pescina 


\section{APRILE 2018}

09:30 - 11:30 SECONDA SESSIONE PLENARIA

Prospettive di applicazioni dopo le ultime note giurisprudenziali

Aula Magna della legge Gelli-Bianco e le sue reali applicazioni

PRESIDENTI

Enrico Marinelli, Simona Zaami

Giovanni Battista Petti, Presidente della III Sezione Civile della Suprema Corte di Cassazione

Enrico Pedoja, Segretario Società Medicolegale Triveneta

Presentazione del Dizionario Italiano del Crimine di Vincenzo Maria Mastronardi, a cura del Dott. Stefano Loparco, Direttore generale Osservatorio Nuove Professionalità (ONP)

Break

11:45 - 12:30 Responsabilità sanitaria - Aspetti penalistici

Aula Magna

PRESIDENTI

Gianluca Montanari Vergallo, Maria Agnino

1. La responsabilità dell'odontoiatria dopo la legge Gelli-Bianco Fabio Cicchitelli

14:30 - 17:30 TERZA SESSIONE PLENARIA

Standard Internazionale delle tecniche investigative e relativi profili

Aula Magna

PREsidente

Vincenzo Mastronardi

Gregory Vecchi

L'analisi predittiva dei comportamenti: il Profiling dell'aggressore prima di eventuali attacchi

nelle scuole e negli ambienti lavorativi

Claudio Rubertà, Comandante nucleo Radio Mobile di Roma, Italy

Gioacchino Angeloni, già comandante provinciale della Guardia di Finanza di Torino attuale ufficiale di collegamento tra il Comando generale della Finanza e il Ministero del Tesoro

Break

WORKSHOP N. 2

17:45 - 19:00 Scena del crimine

Aula Magna

PRESIDENTE

Giovanni Neri

1. Scena del crimine virtuale: stalking e ghosting Giovanni Cicchitelli

2. Scena del crimine e procurato allarme Michelangelo Russo

3. La grafologia sulla scena del crimine Anna Rita Guaitoli, Giuseppe Marano

4. Il criminal profiling attraverso l'analisi della grafia Monique Iandolo

5. Against terrorism. La conoscenza è potere. Il contributo del criminologo Simona Laura

6. Analisi del fenomeno terrorismo internazionale Maria Ligorio

WORKSHOP N. 3

17:45 - 19:00 Le perizie grafiche ed altri argomenti criminologici

Aula Parallela

PRESIDENTE

Vincenzo Mastronardi

1. Il Contributo della Teoria Stratigrafica applicata alle Scienze Forensi Alessandro Massimo

2. Caravaggio genio e criminale: uno studio grafologico Elena Marchetti

3. La giustizia riparativa nella prospettiva vittimologica integrata Art. 162-ter c.p. Martina Vallesi

4. Disney: il crimine nelle favole Marta Senesi

5. Stalking, malattia mentale, imputabilità, riflessioni su 20 casi Francesca Manunza, Giampaolo Pintor 


\section{APRILE 2018}

09:30 - 13:30 QUARTA SESSIONE PLENARIA

Aula Magna

La giustizia penale tra il Retributivo e il Garantismo

PRESIDENTI

Vincenzo Mastronardi, Lello Spoletini

Cesare Mirabelli, Presidente emerito della Corte Costituzionale, ex presidente del Csm, Professore emerito Università degli studi di Roma Tor Vergata, Italy

Claudio Zanghì, Emerito Prof. dell'Università di Roma Sapienza, Italy

Ricardo Alvarez, Preside della Facoltà di Diritto Università Maimonides, Buenos Aires, Argentina

Giacinto Siciliano, Direttore della Casa di reclusione di Milano San Vittore, Italy

Gennaro Francione, La nuova epistemologia popperiana del processo penale. Prove forti scientifiche e reductio degl'indizi a congetture processuali. (In ricordo di Ferdinando Imposimato)

Eugenio D'Orio, Specialista in biologia e genetica forense, ricercatore Università di Copenhagen, Denmark; Direttore del Master "Biologia forense ed investigazioni scientifiche" dell'ISF "DNA, elemento spesso over-estimato", Italy 



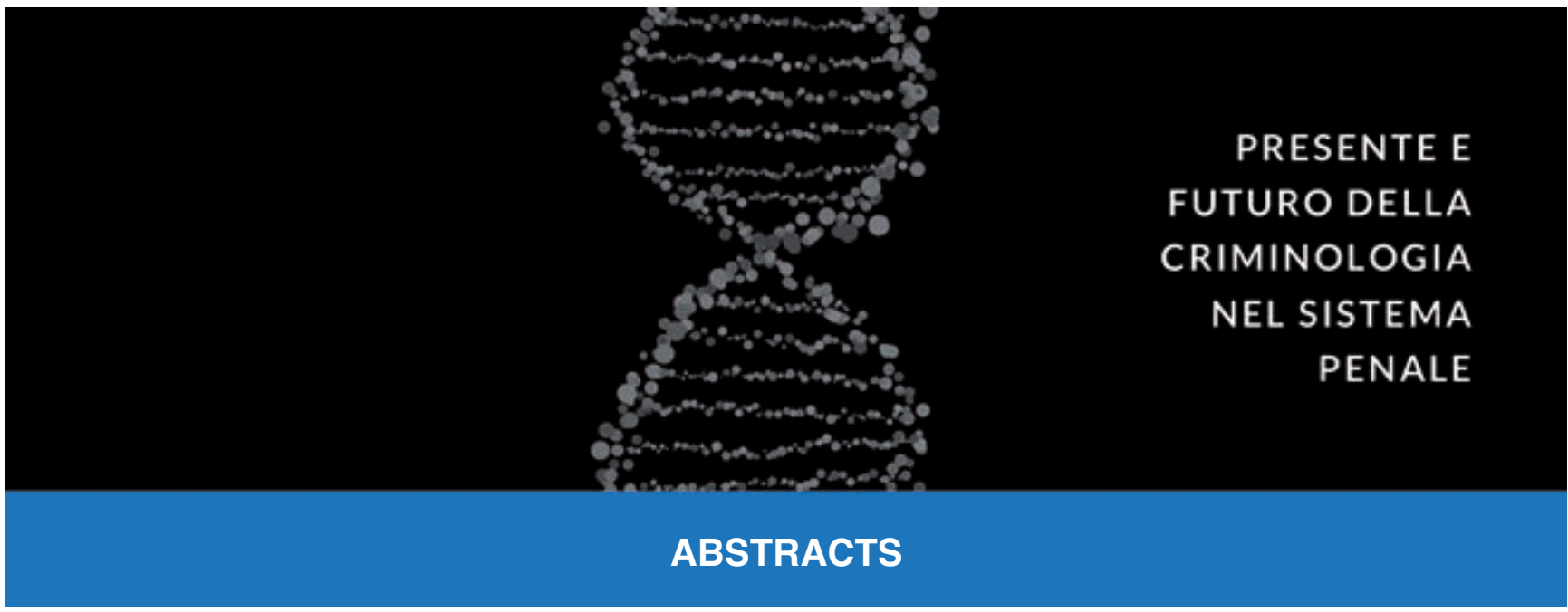

\section{Contributo della Teoria Stratigrafica applicata alle Scienze Forensi}

\section{The contribution of the Stratigraphic Theory applied to the Forensic Sciences}

Massimo Alessandro

Criminologo, Grafologo, Dirigente Medico U.O.M.L. ASL Avellino, Italy

Come sta a riposo un uomo scontroso, maldisposto, un uomo che non vuol avere a che fare con alcuno? Perché gli uccelli possono definirsi più intelligenti dei rettili? Perché un pianista che subisce un insulto apoplettico di una certa portata dovrà dire addio al suo strumento? Perché si “mostrano i denti” in una situazione di eccitazione aggressiva? E perché un cavallo arrabbiato drizza le orecchie? Lo sapevate che Napoleone "mangiava con velocità e precipitazione orrende" e che aveva una stupefacente memoria locale? E ancora perché nel Ballo di San Vito o nel Morbo di Parkinson la scrittura diventa un sintomo patognomonico? A questi ed altri interessanti quesiti cercheremo di rispondere con la nostra relazione che sarà incentrata sulla importanza delle Teorie di Rudolf Pophal applicate alla grafologia ma che come vedremo vanno ad incrociare diversi saperi e proprio per questo diventano nozioni interessanti per diversi professionisti. E lo faremo rivisitando insieme l'opera più importante dell'insigne medico grafologo tedesco che nel 1945 ottenne uno dei pochi insegnamenti universitari dell'epoca (Amburgo) e cioè Die Handschrift als Gehirnschrift, che egli ha dedicato alla moglie Maia, sua collaboratrice. Si tratta, come ebbe a dire Bruno Vettorazzo nella prefazione all'opera tradotta in italiano solo nel 1990, "di un lavoro impegnativo e complesso per dottrina ed erudizione, che fornisce la dotta base filosofica e antropologica al vero profilo grafologico e neuropsicologico". II Contributo della Teoria Stratigrafica che Rudolf Pophal (1893-1966) ha applicato alla Grafologia, travalica a parer nostro i confini della sua originale appartenenza per approdare al più ampio mosaico delle discipline afferenti alle Scienze Forensi quali la Neuropsicologia, le Neuroscienze, la Criminologia Clinica, la Psicologia, l'Antropologia, l'Embriologia, la Biologia e l'Anatomia Comparata. Pophal, allievo di Klages, ne apprese dal maestro la dottrina caratterologica e grafologica e forte della sua formazione accademica neurologica e psichiatrica, ha potuto caratterizzare i suoi studi avendo sentito l'esigenza di una impostazione scientifica del metodo grafologico creando una chiave interpretativa del tutto nuova e originale nell'ambito della Neurofisiologia. Scopo della relazione è fare il punto sull'apporto che tale interessante teoria può dare non solo al campo del grafologo giudiziario ma anche a tutte le menzionate discipline scientifiche partendo dalla sua originale formulazione e anche alla luce dei recenti progressi delle metodiche applicate ai vari campi della ricerca criminologica.

\section{Bibliografia}

Pophal R. (1990), Scrittura e Cervello, La grafologia alla luce della teoria stratigrafica, Ed. Messaggero Padova

Muller W.H., Enskat A. (1995), Diagnostica grafologica, Ed. Messaggero, Padova. Messinger J. (2016), Il linguaggio del corpo rivelato, Ed. PIEMME Blumenfeld H. (2014), Neuroanatomia attraverso casi clinici, Edizioni Piccin Padova. Guyton A.C. (1996), Neurosciences Neuroanatomie et Neurophysiologie, Edizioni Piccin, Padova.

\author{
L'istigazione al suicidio e modalità di estrinsecazione \\ Instigation to suicide and extrinsic modes \\ Francesca Arpino \\ Avvocato, Dottore in Psicologia, Criminologa, Italy
}

La suicidologia è una scienza che si interessa della tutela del bene vita, della prevenzione e del contrasto del suicidio. Le Linee guida, gli strumenti diagnostici e di valutazione che vengono adoperati, hanno contribuito a supportare gli individui in situazione di marcato disagio, laddove fosse alto il rischio di tentativo suicidario. L'acting out suicidario risulta essere una risposta a una sofferenza, a un malessere, si ravvisa nello specifico per l'uomo una carente comunicazione con il proprio es 0 una mancata integrazione con una collettività, diverse le teorie. Per essere perfezionata l'istigazione la condotta partecipativa è tale da determinare con mezzi vari una volontà distruttiva, saldo deve apparire il proposito, la compartecipazione fisica, psichica e il disvalore. Interessante analizzare se l'azione suicidaria derivi da una condotta esterna, tanto da provocare una morte nell'individuo stesso. Con la parola istigazione al suicidio intendiamo nello specifico indicare chiunque rafforzi o determini il proposito suicidario altrui, come parafrasato dall'art 580 c.p agevolando l'esecuzione dell'atto. Ai fini della configurabilità del reato di cui all'art 580 c.p, come cita la sentenza $22780 / 2010$ deve essere evidente la dimostrazione che tale condotta contribuisca con il suo agito il produrre dell'evento morte. Fra le altre caratteristiche che possono ravvisarsi deve essere presente una dipendenza fra autore del reato e suicidio in termini di azione e reazioni, considerando nella dinamicità degli eventi il dolo e la casualità. La fenomenologia del reato deve riconsiderare una serie di aspetti, per questo reputo opportuno citare un caso concreto, avvenuto nella contea di Fair haivens. Nella fattispecie presa in esame, si vede come protagonista una donna, Michelle Carter, con il suo compagno Conrad Roy. Dall'analisi dei fatti, all'interno della coppia emergeva il quadro di una relazione disfunzionale, un rapporto platonico che poteva trovare con il perfezionamento del suicidio la liberazione di un profondo dolore e una profonda frustazione. II legame della coppia fa trasparire una dipendenza all'interno di un quadro patologico di ambedue i soggetti. Un profondo pessimismo contraddistingueva Conrad, e vari disturbi soprattutto di ordine alimentare erano presenti in Michelle. Vero che Conrad Roy venne trovato morto la notte del 12 luglio del 2014, la causa dell'evento fu un avvelenamento di monossido di carbonio, avvenuta all'interno del suo pick-up. La compagna ancora minorenne venne formalmente accusata di omicidio colposo. Dalla ricostruzione degli eventi, pare che la medesima avesse con una serie di messaggi incitato il giovane a porre fine alla propria vita. Dalla lettura dei messaggi di quella notte non si evince nessuna chiamata ai soccorsi, nonostante Conrad Roy aveva reso manifesto il suo proposito suicidario. Altresi dalla lettura degli sms appare chiaro come Michelle con una serie di frasi invita l'uomo a compiere l'estremo gesto: "Sei pronto? Devi solo farlo: Non puoi ignorare la questione. Quando smetti di ignorare la domanda? Non puoi continuare a d evadere la questione. Bata andare da qualche parte con il pick up “.........." E' il momento giusto e tu sei pronto. e allora fallo bene...tra le altre parole che si leggono notiamo... rientra in quella macchina hai bisogno di farlo. Tutto quello che hai bisogno di fare." Tra i due intercorsero 20000 messaggi, tra le tante conversazioni emergeva 
la volontà di Roy di non lasciare la famiglia. I vari agiti della donna furono interpretati dagli investigatori come atti per occultare le prove che palesavano l'avvenuto suicidio. La difesa di Michelle chiese di archiviare il processo all'appellandosi alla libertà di parola (www.ilmessaggero.it di Federica Macagnone 7/6/2017). Le prove raccolte nel 2015 erano divenute materiale non solo pubblico, ma valido ai fini processuali, contro l'accusa di involontary manslaughter. La tesi difensiva nei confronti di Michelle faceva emergere elementi che avvaIoravano un reale istinto di morte di Conrad (furono evidenziati durante il processo alcuni sms ove si leggeva "facciamo come Romeo e Giulietta"). II dubbio che dominò in sede giudiziale, (considerando le varie frasi scritte da Michelle allo stesso) era: Possono le parole diventare un'arma? L'avvocato Cataldo, richiamando il primo emendamento della costituzione degli Stati Uniti, evidenziando il diritto della Parola e di Stampa tentò la difesa di Michelle, che però venne condannata a titolo di omicidio colposo per aver indotto il suicidio. La sentenza del giudice Moniz fu considerata esemplare perché responsabilizza i soggetti nel loro comportamento virtuale. Sentenza questa, che auspica un intelligente utilizzo della rete e dei sistemi informatici, affinchè non possano essere presenti prevaricazioni o vessazioni nel social, considerando quanto l'aggressività verbale possa colpire e ledere diritti e le persone fino a deteriorare la psiche e fino a causare danni e eventi imprevedibili. Michelle Carter è stata condannata a 30 mesi di condanna, da scontare tra reclusione e libertà condizionata. Le varie interpretazioni suicidarie possono essere ravvisabili anche attraverso la letteratura. La letteratura infatti è costellata da opere che ricordano come anche le condotte esterne possano modificare la vita degli individui: i) L'Antigone: un suicidio reso per un'ingiustizia subita in nome di un affetto e un valore; ii) Madame Butterfly: una morte procurata, sopraggiunta dopo il desiderio e l'attesa di ricongiungersi a un compagno dopo la nascita di un figlio; iii) Madame Bovary: un suicidio maturato dopo una depressione e una vita di adulterio; iv) I dolori del giovane Werther: un suicidio che nasconde un amore non corrisposto, testo questo che venne anche bloccato per non aumentare il rischio suicidario, cercando di non mettere in correlazione amore e morte; v) Le ultime lettere di Jacopo Ortis: un suicidio dietro a un ritiro sociale. Lo studio dei vari segnali, i test, la decodifica visiva sono un primo passo per contrastare un alto rischio suicidario,e rappresentano un valore aggiunto per comprendere nella fenomenologia tutti quegli elementi di aggressività che comportano il verificarsi di eventi drammatici.

\section{Bibliografia}

Aportone A. (2003), Sull'etica del suicidio. Dalle riflessioni e Lezioni di Immanuel Kant con I preparativi di un infelice alla morte volontaria. Di Anonimo del settecento. Editore Le lettere Collana I Biancospini.

Durkeim E. (2014), Il suicidio e studi di sociologia, Bur Biblioteca Univ. Rizzoli.

Pompili M., Tatatrelli R. (2008), Il suicidio e la sua prevenzione.

Sitografia:

Macagnone F. (2007) Usa, Ventenne istiga il fidanzato con centinaia di sms. In http:/www.il messagero.it/amp/usa-20-istiga.fidanzato-al-suicidio-centinaia-disms-accusat-di-omicidio2488978

Danna S. (2017) Usa, Michelle condannata per il fidanzato suicida: l'ha ucciso via sms. In http://www.corriere.it/cronache.it 17 giugno-17/usa-michelle condannata-il-fidanzato-suicida.l'ha-ucciso-via-sms-

La brutta storia di Conrad Roy. In http:/www.il post.it/2016/04/09istigazione-suicidioconrad-roy/amp

www.il messaggero.it di Federica Macagnone (7/06/2017).

\section{Storie maledette. Barbie e Ken, la coppia assassina che ha sconvolto l'Ontario: disturbo psicotico condiviso? Cursed stories. Barbie and Ken, the killing couple who distraught Ontario: Shared psychotic disorder?}

Monica Calderaro

Criminologa Ph.D. Internazionale, Dottore in Psicologia ad Indirizzo Clinico, Grafologa, Perito Grafologo; Docente e Responsabile Didattico nel Corso di Formazione in Grafologia forense "Sapienza” Università di Roma, Docente Master in Criminologia UNINT Università degli Studi Internazionali di Roma; Ricercatore Ist. Internaz. di Scienze Criminologiche e Psicopatologico Forensi - (MIUR codice car n. 62065CSS); Tutor Area disciplinare Criminologica Università degli Studi di Roma Unitelma Sapienza, Italy

II mondo è sicuramente popolato da coppie criminali sia essi catalogati come serial e/o spree killer, decisamente diversi come modus operandi, tuttavia ancor oggi, capita che le due fenomenologie, vengano confuse tra loro. I serial killer che agiscono in coppia, costituiscono tra gli altri, da sempre oggetto di attenzione, soprattutto nella percezione del movente e di come fare ad individuare determinati comportamenti a rischio che indubbiamente portano a generare una sorta di panico sociale soprattutto alla luce dei vari 'modus operandi' che queste coppie mettono in atto, spesso difficili nella loro individuazione, apparendo inizialmente come persone normali. II focus attentivo da parte della comunità scientifica, è mirato oltre alla dinamica che caratterizza la coppia assassina, la motivazione che vi è a monte e che spinge due individui a commettere reati efferati, come nel caso dei coniugi Paul Kenneth Bernardo e Karla Homolka noti come 'Barbie and Ken', i quali apparendo come una coppia di giovani rampanti con un fare disinvolto, sembravano appena usciti da un tipico catalogo della Mattel. Hanno operato nell'area geografica dell'Ontario in Canada, il caso fu portato all'evidenza non solo dalle cronache internazionali, ma anche in diverse pubblicazioni scientifiche, per comprendere proprio la tipologia della dinamica di questa coppia criminale unita dal matrimonio, sebbene come serial killer che agiscono in coppia, rientra in una casistica meno frequente rispetto a quella individuale. Di solito all'interno della coppia, si innesca un comune delirio che li spinge a reiterare in una sorta di cosiddetta 'coazione a ripetere' delle Ioro azioni delittuose, che la inquadrano clinicamente nella nota 'folie a deux ' (Lasègue e Falret fine '800), classificata come 'disturbo psicotico condiviso secondo il DSM-5' e che vede generalmente un soggetto dominante 'Induttore' ed uno debole 'Indotto', i quali nonostante caratterizzati da un universo personologico decisamente diverso, si attraggono tra loro con peculiare ausilio che il cosiddetto soggetto debole fruisce al "delirio omicidiario condiviso' in una relazione patologica in cui entrambi i soggetti esercitano un ruolo specifico. Diversi sono gli autori di ieri e di oggi, che si sono espressi in merito al cosiddetto 'amore malato' che unisce due individui e che nel caso specifico, viene rappresentato dalla coppia unita da un legame matrimoniale che rientra nella categoria di 'coppie miste', mettendo in risalto i diversi fattori che caratterizzano tale tipologia di coppia assassina, con attitudine ad una specifica perversione detta "Ibistrofilia" (vedi John Money, sessuologo Università Johns Hopkins) che dal greco hybrizein commettere violenza contro qualcuno e philia amore, dove uno dei due soggetti, si eccita sessualmente quando il partner commette un atto violento come ad esempio stupro, omicidio etc., ben rappresentata peraltro, nel personaggio interpretato da Faye Dunaway nel ruolo di Bonnie Parker nel film 'Gangster Story'. Interessante si presenta una ricognizione mirata all'analisi comportamentale della suindicata coppia criminale e confrontarla con altre simili, alla luce degli aggiornamenti sul tema.

\section{Bibliografia}

Fornari U., Birkhoff J. (1996), Serial Killer, Centro Scientifico Editore, Torino.

Mastronardi V., Ricci S., Lucchini M., Pomilla A. (2012), Delirio omicidiario condiviso nelle coppie di serial killer, Rivista di Criminologia, Vittimologia e Sicurezza.

Mastronardi V. (2012), Manuale per operatori criminologici e psicopatologi forensi, Edizioni Giuffrè, Milano.

Mastronardi V., De Luca R. (2005), I serial killer. Chi sono e cosa pensano? Come e perché uccidono? La riabilitazione è possibile? Newton \& Compton Editori, Roma.

Michaud S.G., Hazelwood R. (2009), Storie di Perversioni Criminali (Serial Killer e Sadici sessuali), Edizioni Mediterranee, Roma.

Money J. (1986), Lovemaps: Clinical Concepts of Sexual/Erotic Health and Pathology, Paraphilia, and Gender Transposition in Childhood, Adolescence, and Maturity. New York: Prometheus Books. ISBN 0-8290-1589-2.

Kernberg O.F. (2011), The Sexual Couple: A Psychoanalytic Exploration. The Psychoanalytic Review. Vol. 98, Foreshadowing the Present: The Legacies of Theodor Reik, pp. 217-245.

Sighele S. (2008), La coppia criminale, Musso Editori.

\section{La responsabilità dell'odontoiatra dopo la legge Gelli-Bianco Dentistry and malpractice in Italy: The Gelli-Bianco law}

Fabio Cicchitelli

Odontoiatra e Criminologo, Italy

La legge 8 marzo 2017, n. 24, rubricata "Disposizioni in materia di sicurezza delle cure e della persona assistita, nonché in materia di responsabilità professionale degli esercenti le professioni sanitarie", pubblicata in Gazzetta Ufficiale, serie Generale, n. 64 del 17 marzo 2017, ed entrata in vigore giorno 1 aprile 2017, come noto, segue dopo poco più di un quadriennio la legge n. 189/2012 (la c.d. legge Balduzzi). Detto provvedimento normativo, conosciuto anche come legge Gelli-Bianco - dai cognomi dei propositori/estensori Sen. Federico Gelli e Sen. Amedeo Bianco, entrambi medici - ha provato, per l'appunto, a superare i nodi 
problematici emersi nella concreta applicazione giurisprudenziale della legge Balduzzi, nel tentativo di fornire un contemperamento dei contrapposti interessi in gioco: il diritto alla salute, la tutela della dignità professionale e personale dell'esercente la professione sanitaria, il contrasto alla medicina difensiva ed all'incremento della spesa pubblica in materia sanitaria. La legge Gelli-Bianco ha in sé norme relative alla responsabilità penale e a quella civile, nonché profili amministrativi, nella fattispecie attinenti al settore assicurativo. Durante il VI Congresso Nazionale dell'Accademia Italiana di Odontoiatria Legale (Oelle), che si è tenuto nel novembre 2017 a Bologna, odontoiatri, consulenti di parte, avvocati, medici legali e docenti di diritto pubblico e privato si sono confrontati sulla legge dal particolare punto di vista della libera professione e dell'odontoiatria; in tale sede, il Prof. Angelo Riccio, Docente di diritto civile e delle assicurazioni private all'Università di Bologna, ha definito la GelliBianco "una legge spazzatura" e il Prof. Salvatore Oleo, Ordinario di diritto penale e criminologia all'Università di Catania, con espresso riferimento all'art. 5 (relativo alle c.d. linee guida per l'esercente la professione sanitaria), l'ha definita "una norma criminogena" (per il giurista, intervenuto all'ultimo congresso della Società italiana di ortopedia e traumatologia, una disciplina che convive da anni con un altissimo livello di contenzioso medico legale, "Il medico scarso che fa una cosa che non sa fare, potrà dire di aver seguito le linee guida e non sarà punibile"). Non meno severo è stato il giudizio del Prof. Paolo Cendon, uno dei massimi esperti italiani in materia di "nuovi danni", da me intervistato il 15/03/2018: "una legge arrogante, da abbandonare al più presto". Prima di passare alla disamina dell'impatto che la legge Gelli-Bianco ha avuto sulla professione odontoiatrica, giova rammentare l'ultima sentenza delle Sezioni Unite della S.C. di Cassazione n. 8770/2018: per le Sezioni Unite - che hanno risolto il contrasto giurisprudenziale sorto all'interno della Quarta Sezione circa la querelle della permanenza della "colpa lieve" dopo l'avvento della legge Gelli-Bianco - la responsabilità sanitaria in caso di colpa lieve non è stata espunta dal nostro sistema penale. Nei suoi tratti salienti, la responsabilità dell'Odontoiatra dopo la legge Gelli-Bianco, si può così riassumere: i) Mancano ancora le c.d. "linee guida" per l'Odontoiatra (art. $5)$, dato che è controverso possano essere considerate tali le "Raccomandazioni cliniche in odontostomatologia" (390 pp.), emanate nel 2017 dal Ministero della Salute. ii) L'Odontoiatra (nel suo studio) è l'unico su cui ricade la responsabilità come direttore sanitario e come responsabile del processo di sterilizzazione. iii) L'Odontoiatra, in caso di danno iatrogeno, risponde, in base alla I. Gelli-Bianco (art. 7), a titolo di responsabilità civile contrattuale (artt. 1218 e ss. c.c.); dubbi sull'Odontoiatra che lavora per i grandi Centri odontoiatrici, che dovrebbe rispondere per responsabilità civile extracontrattuale (artt. 2043 e ss. c.c., gravando sul Centro quella contrattuale (varii escamotage, tipo cooperative tra odontoiatri, cercano di sovvertire ciò). iv) Ex art. 8 I. 24/2017, sussiste una condizione di procedibilità della causa civile: art. 696-bis c.p.c. (A.T.P. in funzione conciliativa) o mediazione ex d.Igs. 28/2010. v) Per la liquidazione dei danni a carico della struttura e del sanitario si applicano le tabelle del danno biologico ex artt. 138 e 139 del Codice delle assicurazioni private, come modificati nell'agosto 2017 e, di conseguenza, le Tabelle per le lesioni micro e macropermanenti (Tabelle di Milano, modificate nel marzo 2018). vi) Sono nebulosi i dati del contenzioso scaturente dagli eventi avversi in odontoiatria, anche se tutti (SIE, Blog Dentaltrey, De Introna-Tonelli, ecc.) concordano sulla crescita notevole dello stesso negli ultimi anni (non solo in odontoiatria) e la prevalenza del danno da protesi e implantoprotesi, anche se sovente gli interventi contestati sono multipli e difficilmente catalogabili in una tipologia esclusiva; le uniche ad avere dati certi sono le Compagnie Assicurative (contenzioso anche stragiudiziale), che non li divulgano, ma li usano per i loro fini aziendali. vii) Obbligo di assicurazione (art. 10, I. 24/2017), anche se i grandi Gruppi Assicurativi, tipo Allianz, "fuggono"; meglio per gli odontoiatri scegliere polizze con massimali "bassi" (1,5 milioni di euro), ma con clausole che coprano il più possibile, anche l'aspetto della restituzione dell'onorario, che è voce di danno risarcibile, sovente scoperta. A tutt'oggi mancano i decreti attuativi in ambito assicurativo. viii) L'art. 15 della legge Gelli-Bianco prevede che nei processi civili e penali sulla responsabilità sanitaria, il giudice dovrà affidarsi ad un medico specializzato in medicina legale ed a uno o più specialisti nella disciplina oggetto del procedimento, con conoscenza specifica e pratica della disciplina, ma anche competenze nell'ambito della conciliazione. Semaforo rosso, quindi, ai consulenti improvvisati.

\section{Bibliografia}

A.N.D.I., Pro.0.F. (2016), Tabelle di valutazione del danno odontostomatologico, ANDI MEDIA, Roma.

Cortivo P., Betti D., Bordignon D., Favero L. (1990), /l risarcimento del danno in traumatologia dentaria, Piccin Editore, Padova.
De Introna A., Tonelli M. (2014), Casistica in tema di responsabilità odontoiatrica ed aspetti giuriprudenziali, in Tagete 1 - Archives of Legal Medicine and Dentistry.

De Michelis B., Modica R., Re G. (1998), Trattato di Clinica odontostomatologica, Minerva Medica Editore, III Edizione, Torino.

Di Leo D., Tessadri A. (1989), Odontologia forense, Piccin Editore, Padova.

Montagna F. (2005) Testo atlante di odontoiatria medico-legale, Masson Editore, Milano.

Norelli G.A., Pinchi V. (2010), Odontologia Forense, Piccin Editore, Padova.

Umani Ronchi G. (1993), Medicina Legale in Odontostomatologia, Lombardo Editore, Roma.

Umani Ronchi G., Mastronardi V., Sommazzi A. (2008), Odontoiatria Forense, Libreria Cortina Editore, Torino.

\section{Scena del crimine virtuale: stalking e ghosting Virtual crime scene: Stalking and ghosting}

Giovanni Cicchitelli

Avvocato del Foro di Cosenza e Criminologo, già Cultore di Diritto Privato presso I'Università degli Studi della Calabria, Italy

La scena del crimine è lo spazio fisico territoriale, concettuale e virtuale nel quale si è svolto il crimine. Sovente, nel delitto di cui all'art. 612bis c.p., rubricato "Atti persecutori" (c.d. stalking), la scena del crimine è (anche 0 solo) virtuale, poiché tale reato può essere commesso (anche o solo) in maniera, per così dire, "tecnologicamente assistita" (si pensi, palesemente, all'uso dei social media e di internet in generale). II delitto di "atti persecutori" (o stalking, dall'inglese to stalk, letteralmente "fare la posta") è stato introdotto nel sistema penale italiano mediante il d.I. n. 11/2009 (convertito, con modificazioni, nella I. n. 38/2009), che ha inserito nel Codice Penale, appunto, l'art. 612-bis; la norma ha poi subito altre modifiche nel 2013. Dalla rassegna della giurisprudenza, specie della S.C. di Cassazione (cfr., ex plurimis, Cass. n. 17082/2015), se ne ricava che trattasi di un "reato abituale di evento, a struttura causale e non di mera condotta", che si caratterizza per la produzione di un evento di "danno" (consistente, cioè, in alternativa, nell'alterazione delle abitudini di vita, 0 in un perdurante e grave stato di ansia o di paura, ovvero, di un fondato timore per l'incolumità propria, di un prossimo congiunto o di una persona alla quale il soggetto è legato da relazione affettiva); gli eventi contemplati dalla norma e tra Ioro alternativi devono essere in rapporto di immediata causalità con la condotta di aggressione. Altri aspetti del delitto de quo sono ricavabili dai seguenti arresti giurisprudenziali della Cassazione, cui si rinvia per brevità: n. 14997/2012; n. 45648/2013; n. 46331/2013; n. 17082/2015; n. 29859/2015; n. 21407/2016; n. 574/2017; n 14462/2017; n. 49216/2017; n. 104/2018, ecc.). L'introduzione dello stalking quale figura autonoma di illecito penale ha destato non poche perplessità in seno alla dottrina penalistica, a causa della tecnica normativa adottata, non scevra di una qualche indeterminatezza nella descrizione della fattispecie. Personalmente, sono affezionato al "Rasoio di Occam": Entia non sunt multiplicanda sine necessitate, mentre talvolta si creano reati per finalità extragiuridiche e simbolicoespressive, socialmente tranquillanti, ma in pratica dal funzionamento difettoso. A conferma di ciò, molti o forse quasi tutti i "laici" (cioè coloro che non sono operatori del settore) sono sovente convinti di "conoscere" il reato di stalking, o magari il fenomeno del mobbing in ambito lavorativo, dato che tra certe trasmissioni televisive e internet è facile accedere ad informazioni di ogni tipo, spesso aberranti e/o fuorvianti, come accade pure per la medicina, con l'effetto che il quisque de populo si persuade di essere stalkizzato, mobbizzato o ammalato, portando all'esasperazione il più stoico degli operatori del settore con convinzioni perniciose e/o difficili da eradicare. Ciò doverosamente premesso, dopo l'abuso del delitto di stalking, di recente si è individuata una nuova "modalità comportamentale malata di coppia": il ghosting, neologismo coniato dai media statunitensi per "scolpire" il trattamento che la bellissima Charlize Theron avrebbe riservato all'ex compagno Sean Penn: sparire come un fantasma, come se nulla fosse mai stato o esistito; di fatto, si tratta di una tattica interpersonale passivo-aggressiva, con regole spietate: sparire, non dare spiegazioni, negarsi e comportarsi vigliaccamente, come se il partner fosse un perfetto estraneo. Un vero e proprio capolavoro di vigliaccheria, se si pensa che bloccare la comunicazione tramite telefono, social media, internet, ecc., è estremamente facile e deresponsabilizzante, poiché evita il confronto diretto, de visu et de auditu; ad aggravare il senso di mortificazione dell'ex partner, ci si mettono i social, che pongono, volenti o nolenti, al cospetto della vita dell'altro che - talvolta forse solo in apparenza e/o per senso di rivalsa - prosegue come nulla fosse mai stato, magari iniziando pure una nuova liason in tempi ridottissimi. La vittima (perché a mio avviso di vit- 
tima trattasi) del ghosting rischia, viceversa, di passare per uno stalker, dato che vorrebbe ricevere delle spiegazioni, ma viene trattato ex abrupto come un estraneo invadente, che si prende delle "confidenze" con l'ex partner (sic!), pur avendo con questo condiviso un segmento, più o meno lungo ed importante, della propria esistenza. In conclusione, di fronte a complessi di condotte che mi piace definire "alla Giano bifronte" (cioè che, se viste dall'angolazione della presunta vittima, potrebbero parere stalking, mentre, se guardate dall'ottica dell'ipotizzabile autore del reato, potrebbero invece essere solo una reazione all'altrui ghosting), è sempre auspicabile un atteggiamento di somma attenzione della Magistratura, sia requirente che giudicante.

\section{Bibliografia}

Cassano G. (2017), Stalking, atti persecutori, cyberbullismo e tutela dell'oblio, Wolters Kluwer, Assago.

De Simone G. (2013), I/ delitto di atti persecutori, Aracne, Roma.

Edwards S. (2016), Charlize Theron Broke Up With Sean Penn By Ghosting Him, Jezebel. Liberali B. (2012), II reato di atti persecutori. Profili costituzionali, applicativi e comparati, Franco Angeli, Milano.

Micoli A. (2012), Il fenomeno dello stalking. Aspetti giuridici e psicologici, Giuffrè, Milano.

Safronova V., (2015) Exes Explain Ghosting, the Ultimate Silent Treatment, The New York Times.

Sarno F. (2010), Il nuovo reato di atti persecutori (art. 612-bis), Giuffrè, Milano.

Tasciotti U., Bruno A. (2014), Lo stalking dall'origine, Aracne, Roma.

Tovani S., Trinci A. (2013), Lo stalking. II reato di atti persecutori: aspetti sostanziali e processuali, Dike Giuridica Editrice, Roma.

\section{La grafologia sulla scena del crimine Graphology at crime scene}

Anna Rita Guaitoli, ${ }^{1}$ Giuseppe Marano ${ }^{2}$

${ }^{1}$ Grafologa, Giornalista, Psicologa; ${ }^{2}$ Grafologo, Perito Grafogiudiziario, Italy

In una realtà complessa come quella odierna, nella quale sempre più forti si avvertono le conseguenze dell'anomia familiare e sociale, è più facile che i giovani passino da comportamenti a rischio a comportamenti devianti: la linea di confine tra normalità e patologia si è fatta ormai veramente sottile. I delitti familiari sono sempre esistiti, ma se prima erano rari tanto da interessare la narrazione di più generazioni, oggi sono, con evidenza, all'ordine della cronaca. La grafologia è una scienza umana che legge il tracciato grafico, interpretando i segni che vi compaiono messi in relazione dinamica tra loro. D'altro canto la scrittura è comportamento sociale dal momento che riflette le dominanti culturali del tempo; ogni elemento grafico andrà perciò verificato, in una analisi anche quantitativa. II tracciato grafico è, altresì, espressione personale delle forze evolutive che modellano la personalità, di volta in volta segnando il percorso individuale verso la conquista dell'identità. Ė su questo processo, su questa trasformazione, che interviene il lavoro del professionista grafologo. Si procede dunque all'interno di un contesto grafico in cui i segni messi in relazione tra loro sulla base dei quattro elementi costituivi di ogni grafismo (tratto, movimento, spazio, forma) permettono di evidenziare le forze e le controforze, i bisogni e le motivazioni, unici di quella persona. Nel caso specifico: non esiste il "segno dell'assassino". L'intervento grafologico si potrà invece a sviluppare su due piani. Nella fase definibile di profiling, l'analisi dello scritto coevo 0 il più possibile vicino all'evento criminale, può aiutare a tracciare tratti della personalità che potranno incontrare i risultati di altre analisi, rafforzandoli o contrastandoli. Si possono, ad esempio, individuare nel tracciato grafico: uno stile di comportamento impulsivo con conseguente difficoltà a controllare gli impulsi; uno stile di comportamento riflessivo di tipo "ruminante" con tendenza ad accumulare rabbia senza trovare opportuno sfogo delle emozioni; la costruzione di una maschera comportamentale in cui sarà possibile rilevare cedimenti grafici o eccessive contraddizioni; il livello delle competenze emotive, una condizione affettività sofferente e/o immatura, la carenza di autostima. Nella fase di analisi longitudinale, possibile con scritti che coprano diverse età dello scrivente, si potrà evidenziare il percorso che ha portato a quella scrittura, identificando il momento in cui la dinamica costruttiva abbia subito un arresto, la carenza di competenze emotive personali e/o di competenze sociali. La famiglia rappresenta un territorio sicuro all'interno del quale è possibile trovare riparo dalle problematicità e dalle sollecitazioni dell'ambiente esterno. Un luogo in cui tutti i componenti del nucleo familiare dovrebbero scambievolmente accogliersi, consolarsi ed incoraggiarsi; promuovendo il benessere della persona e la costruzione di un adeguato legame di attaccamento. Tuttavia, può accadere che il contesto familiare non riproduca più il grembo in cui si sviluppa un soddisfacente modello interiore di relazioni con l'altro da sé, ma finisca per configurarsi come il grembo dove si verificano azioni criminose, spesso spietate e ad elevato impatto emozionale: i figli che uccidono i propri genitori, per esempio. Diversi autori si sono dedicati all'analisi di questo fenomeno, le complesse ed articolate ragioni che spingerebbero ad assassinare i propri genitori sembrano essere connesse con i comportamenti violenti di cui i bambini possono essere vittime o testimoni, unitamente a tratti disfunzionali di personalità che sovente caratterizzano gli autori di matricidio e parricidio. Nell'ambito dei potenziali indici predittivi di delitti compiuti contro i propri genitori, Bourget e collaboratori segnalano la disorganizzazione dei comportamenti e un significativo peggioramento di una preesistente malattia psicotica, Calvete et al. hanno individuato negli aspetti di un narcisismo patologico un fattore prognostico di aggressività dei figli nei confronti dei genitori.

\section{Bibliografia}

Boille N. Il gesto grafico gesto creativo. Trattato di grafologia. Borla, Roma, 2006.

Bourget D., Gagné P., Labelle M.E. Parricide: a comparative study of matricide versus patricide. J Am Acad Psychiatry Law. 2007;35(3):306-12.

Bowlby J. Attaccamento e perdita. Vol. 2: La separazione dalla madre. Bollati Boringhieri, Torino, 2000.

Calvete E., Orue I., Gamez-Guadix M., Bushman B.J. Predictors of child-to-parent aggression: A 3-year longitudinal study. Dev Psychol. 2015 May;51(5):663-76.

Crépieux Jamin J. ABC della grafologia. Edizioni Messaggero, Padova, 2001.

Dantas S., Santos A., Dias I., Dinis-Oliveira R.J., Magalhães T. Parricide: a forensic approach. J Forensic Leg Med. 2014 Feb;22:1-6.

Freud S. Al di là del principio di piacere. Mondadori, Milano, 2007.

Grattagliano I., Greco R., Di Vella G., Campobasso C.P., Corbi G., Romanelli M.C., Petruzzelli N., Ostuni A., Brunetti V., Cassibba R. Parricide, abuse and emotional processes: a review starting from some paradigmatic cases. Clin Ter. 2015;166(1):e47-55.

Guaitoli A.R., Manetti E. Identità, scrittura e segni. Percorsi evolutivi tra teoria e tracce segniche dall'infanzia all'adolescenza. Pioda Imaging, Roma, 2005.

Guaitoli A.R. Accogliere la fatica di chi cresce. seguendo la traccia del segno grafico. Epsylon, Roma, 2015.

Mastronardi V. Manuale per operatori criminologici e psicopatologi forensi. Giuffrè, Milano, 2012

\section{II criminal profiling attraverso l'analisi della grafia Criminal profiling by handwriting analysis}

Monique landolo

Criminologa, Grafologa Giudiziaria, Socio Ordinario A.G.I., Italy

Nell'atto dello scrivere, ognuno di noi 'proietta' - sia pure a livello inconscio - se stesso sul foglio di carta, lasciando così l'impronta della propria personalità, definita appunto come "personalità grafica" (http://gnosis.aisi.gov.it/Gnosis/Rivista3.nsf/ServNavig/19\#(5). II "profilo grafologico di personalità" si basa sull'analisi minuziosa della impronta grafica dello scrivente, attraverso lo studio di svariati fattori che interagiscono tra loro come, ad esempio, l'occupazione dello spazio della superficie scrittoria, l'armonia, la formazione delle lettere, la modalità con cui il gesto grafico incede, la forza pressoria che viene sprigionata di volta in volta, costanti, variabili e gesti peculiari. Un caso emblematico di come la grafologia forense possa interconnettersi con la criminologia risiede, sicuramente, nel caso del "Mostro di Foligno", autodefinizione posta in essere da Luigi Chiatti, assassino di Lorenzo Paolucci (13 anni) e Simone Allegretti (4 anni) nei primi anni '90. La dottoressa Isabella Zucchi venne incaricata, in qualità di grafologa psicologa, dalla Procura di Perugia, di redigere una perizia grafologica al precipuo scopo di analizzare delle firme apposte su due biglietti firmati "Il Mostro". Nella perizia verrà evidenziata dalla Zucchi, attraverso combinazioni di categorie specifiche grafiche (tra le quali curva angolosa, dimensione verticale e larghezze, inclinazione, gesti fuggitivi...), una personalità "instabile, eccitabile e reattiva" caratterizzata da "congestioni emotive inclini alla depressione" e da "un'ossessione sul piano degli istinti" di un soggetto che porta con sé "pesanti ed arcaici problemi affettivi" (cfr. bibliografia). II profilo di personalità della Zucchi si rivela, dopo la cattura di Chiatti, inquietantemente corrispondente. Di grande interesse è anche lo studio della Zucchi circa la frequenza della presenza di determinate caratteristiche grafiche tra le grafie di un campione di detenuti, quali l'alterazione della concezione del proprio sé, la difficoltà a gestire l'emotività, l'impulsività disordinata, le tendenze mitomani ed il pensiero carente di oggettività nel valutare le cose. Più ci avviciniamo 
al terreno dei crimini violenti e più è necessario fare le dovute precisazioni e differenziazioni: ogni tipologia di crimine contiene al suo interno la presenza di specifiche caratteristiche attraverso le quali è stato possibile definire e classificare Serial Killer, Spree Killer, Stupratori, Pedofili, Piromani, Mass Murderer, Terroristi ed altre classificazioni effettuate da Mastronardi V (cfr. bibliografia). Se partiamo, ad esempio, dall'assunto che la maggior parte dei Serial killer sono caratterizzati tra gli altri, da un tratto di personalità di tipo narcisista e rileviamo la presenza di segni grafici che, solitamente, si ritrovano in questa tipologia di soggetti (come scrittura vigile e tesa, pressione estremamente forte, angoli, zona superiore enfatizzata, colpi tesi, congelamento e spazi estremamente ampi tra le parole), interessante sarebbe studiare campioni significativi di scritture delle diverse personalità criminali suindicate, come già effettuato da Calderaro di cui tra gli altri, anche sugli assassini seriali (cfr. bibliografia). Ciò a conferma della sussistenza di specifiche caratteristiche evinte dalla disamina della grafia (che fa da strumento efficace nell'ambito della criminologia), nella individuazione di un determinato tratto di personalità, cogliendo quelli che potrebbero rivelarsi i cosiddetti ‘segnali di rischio'.

\section{Bibliografia}

Calderaro M. In tema di Serial Killer. Disamina Psicologica dalla Scrittura. In: Rassegna di Psicoterapie. Ipnosi. Medicina Psicosomatica. Psicopatologia forense. Volume 20 - N² 2 maggio - Agosto 2015 Sapienza Università di Roma (Dir. Prof. V. Mastronardi).

Mastronardi V. (2012), Manuale per operatori criminologici e psicopatologi forensi, Edizioni Giuffrè, Milano.

Mastronardi V., Bidoli S.A., Calderaro M. (2010), Seconda edizione Grafologia Giudiziaria e psicopatologia Forense (Metodologia di indagine nel falso grafico e la capacità di intendere e di volere dalla grafia. Giurisprudenza).

Mastronardi V., De Luca R., (2005), I serial killer. Chi sono e cosa pensano? Come e perché uccidono? La riabilitazione è possibile? Newton \& Compton Editori, Roma. Moretti G. (1985), Trattato di grafologia, Padova, ed. Messaggero.

Palermo G.B., Mastronardi V. (2005), II Profilo Criminologico (Dalla scena del crimine ai Proficli Socio-Psicologici), Edizioni Giuffrè, Milano

Zucchi I. (1998), Grafologia della devianza, a cura dell'A.

\section{I vissuti emotivi della vittima durante e dopo l'abuso sessuale infantile}

\section{The victim's emotional experiences during and after child sexual abuse}

Daniela Labattaglia

Psicologa Clinica, Specializzanda in Criminologia Clinica, Psicopatologia e Scienze Forensi; Master di II livello presso I'Università LUMSA di Roma, Italy

L'abuso sessuale destabilizza il funzionamento cognitivo ed emotivo di chi lo subisce poiché le azioni dell'adulto determinano una compromissione psichica in cui si instaura una confusione tra tenerezza e sessualità perversa. All'interno di questo stato di confusione, generato dal bisogno innato di amore, sicurezza e conforto, si innesta nella vittima l'ingenuità di lasciarsi coinvolgere in tali atti senza comprenderne il senso e il significato. Comincia a pensare che le molestie di cui è oggetto siano soltanto dei gesti d'amore e che non ci sia nulla di male; ha trovato ciò di cui ha bisogno nella persona che gli sta rivolgendo attenzioni: il suo abusante. Questa ricerca di amore e l'illusione di averlo trovato diventa la sua condanna, in quanto non possiede strumenti cognitivi ed emotivi adatti a fronteggiare la situazione. Quella che sta subendo, è un'esperienza intrisa di paura, ansia, violenza che non avrebbe dovuto scoprire e tantomeno subire; è un'esperienza che ha derubato la sua innocenza costringendola a diventare grande senza volerlo. La vittima di abusi sessuali si sente contaminata, sporca, umiliata; sente l'ambiguità delle attenzioni sessuali dell'adulto e prova vergogna per essere stata oggetto di desideri sessuali che non è riuscita ad evitare 0 a fermare. L'essere stata vittima di una prevaricazione psicologica e fisica conduce all'interiorizzazione di un Sé debole, ad una sensazione di svalutazione che avvia la vittima ad un processo di disistima, ad una percezione di fallimento e ad un'attribuzione di responsabilità verso se stessa: artefice e poi complice di un gioco per grandi a cui non doveva avvicinarsi e partecipare. L'attribuzione di colpa a se stessa viene alimentata dalle azioni e verbalizzazioni del suo abusante ("sei stata tu ad istigarmi", "pensavo ti piacesse") che fanno precipitare la vittima in un ulteriore stato di impotenza, poiché l'abuso non solo è stato causato, ma è stato anche permesso e non fermato. Inoltre, l'attribuzione a se stessa di essere stata causa dell'abuso serve alla vittima anche per trovare un senso a ciò che è successo e per accettare in qualche modo la realtà di quello che le è stato fatto. Difatti, la domanda che permane per tutta l'esistenza di chi ha subito degli abusi sessuali è "perché proprio io?", una domanda che non riesce a trovare risposta quando si è invischiati in una relazione marchiata da perversione. Per avere una temporanea risposta, la vittima rafforza l'attribuzione di colpa a se stessa e la percezione di essere cattiva, indegna e, di conseguenza, meritevole di tale trattamento. Ma, nonostante l'illusoria soluzione, questa sarà una delle domande che accompagnerà la sua intera esistenza e a cui cercherà di trovare una risposta per tutta la vita. L'abuso sessuale, dunque, è un fenomeno complesso che non tende a risolversi spontaneamente se non vengono attivate adeguate misure di cura e protezione. II dolore silente e non elaborato, legato all'esperienza dell'abuso sessuale, tenderà a cronicizzarsi e a trovare sfogo in manifestazioni fisiche e comportamentali patologiche sia nell'immediato che in età adulta. Pertanto, per evitare che la ferita dell'abuso sessuale resti "sanguinante" all'interno della vita psichica della vittima, è necessario un intervento sinergico e concreto tra le varie figure professionali specializzate nel settore. Risulta fondamentale, laddove sia possibile, individuare tempestivamente la presenza di un presunto abuso sessuale tramite la raccolta di evidenze oggettive, da cui si avvierà un intervento di tutela della vittima e un procedimento penale nei confronti del presunto colpevole. Inoltre, è di fondamentale importanza accogliere la rivelazione della vittima facendo da contenitore ai suoi racconti e a quel groviglio di sentimenti che l'esperienza dell'abuso ha portato con sé. Aiutare una vittima di abuso sessuale significa aiutarla ad elaborare i vissuti di impotenza e le distorsioni cognitive ed emotive sia personali che relazionali e a ridimensionare i meccanismi di difesa disfunzionali (messi in atto come illusione di autoprotezione) per evitare che si adagino su forme di adattamento patologico. Aiutare una vittima di abusi sessuali significa aiutarla a liberarsi da quell'ingombro doloroso e pericoloso che le grava sul cuore.

\section{Bibliografia}

De Leo G., Petruccelli I. (2002), L'abuso sessuale infantile e la pedofilia. Intervento sulla vittima, Franco Angeli, Milano.

Malacrea M. (1998), Trauma e riparazione, Raffaello Cortina Editore, Azzate (Varese). Finkelhor D., Browne A. (1985), The traumatic impact of child sexual abuse: a conceptualizzazion, American Journal of Orthopsychiatric, 55 (4), 530-541.

Burgess A.W., Holmstrom L.L. (1974), Rape trauma syndrome, American Journal of Psychiatry, 131, 981-985.

\section{Against terrorism. La conoscenza è potere. II contributo del criminologo \\ Against terrorism. Knowledge is power. The contribution of criminology}

Simona Laura

Medico, Criminologa, Italy

"Si definisce terrorismo l'insieme delle azioni compiute nell'ambito di lotte armate intese non solo a colpire le forze armate avversarie, ma a diffondere paura, disagio e insicurezza tra le popolazioni civili. II terrorismo è stato da sempre usato per indebolire resistenza e volontà dei propri nemici." II terrorismo islamico ha un origine recente. Dobbiamo premettere che l'uso della parola islamico è di uso corrente, ma la corretta terminologia sarebbe terrorismo islamista. Nasce da un'ideologia, nella sua accezione più estrema, ed in questa ideologia trova la giustificazione maggiore per compiere atti terroristici. II nucleo dunque è la religione islamica fondamentalista che giustifica, incita e sostiene il Jihad. Cosa vuol dire Jihad? Sforzo teso verso uno scopo. II Corano indica 4 modi per condurre al Jihad: i) combattere con il cuore preceduta da una purificazione di sé contro il peccato; ii) combattere con la lingua per predicare la giustizia; iii) combattere con la mano nella punizione del peccato; iv) combattere con la spada per insegnare agli infedeli. Cosa vuol dire Sharia (la via) e Khalifa (Califfo) e shi'a (partito)? La tradizione, cioè la Sunnah, come viene interpretata dagli Ulama (sapienti)? Come viene descritto dagli Imam (Imam vuol dire colui che precede) l'avvento del Mahadi (il Guidato)? Come la Criminologia può essere di supporto? Intanto analizzando le modalità operative, dunque studiando la propaganda (passaggio centrale del fenomeno jihadista) per eventualmente dare uno spunto su quali potrebbero essere le strategie della contropropaganda. La propaganda, nodo fondamentale della cyberwar operata dal terrorismo islamista, deve essere studiata come propaganda alle masse (tramite web e case di produzione), ai piccoli gruppi (nelle moschee durante le prediche e nelle scuole con i programmi scolastici rimaneggiati e destrutturati) e a tu per tu nel processo di reclutamento e radicalizzazione. Interessante 
prendere visione dei processi di training e di brain washing che vengono adoperati sul soggetto radicalizzando per portare a termine l'opera di radicalizzazione. Ed interessante può essere analizzare l'arsenale dell'Isis, la provenienza di esso e la provenienza delle munizioni. Se l'analisi dei flussi economici può portare ad individuare degli illeciti, l'analisi dei flussi mentali può portare all'individuazione, in questo caso, del potenziale jihadista, del reclutando o del radicalizzato? Chi può essere e dove potrebbero essere i reclutatori? Sono persone dedicate al reclutamento e alla radicalizzazione con conoscenze specifiche o sono persone improvvisate? Da cosa si evince la risposta e per quale motivazione? E chi sono le persone a rischio radicalizzazione? Chi potrebbero essere le persone già introdotte? E le persone già introdotte quale comportamento hanno? $\mathrm{E}$ dove si trovano 0 dove si potrebbero trovare 0 dove pensano di potersi ritrovare? E come si aggregano? E in base alla loro ideologia come potrebbero organizzarsi e cosa potrebbero fare? E come lo potrebbero fare? Insomma scopo di questo lavoro vuole essere provare ad entrare nella mente del terrorista islamico o del potenziale terrorista. In questo delicato compito sarà essenziale analizzare il profiling del terrorista, tracciare i luoghi con profiling geografico, avere ogni tipo di informazione con profiling sociologico e culturale, capire il linguaggio, entrare il più possibile nella cultura jihadista, valutare il tipo di soggetto radicalizzabile, avere ogni tipo di informazione sul soggetto radicalizzato, considerare il ruolo delle donne e la motivazione al loro coinvolgimento, valutare la schiavizzazione e il brainwashing sui bambini e sugli adulti. Insomma provare ad entrare nel delirio che sorregge la militanza dell'ISIS e delle organizzazioni terroristiche affini.

\section{Bibliografia}

Albanese A., Gangiulo G., Molle E., Scigliano, Valdenassi E. Daesh Matrix AGC Communication. Settembre 2016

Albanese A., Gangiulo G., Molle E., Baretski R., Balkan G., Valdenassi E. The Islamic state. Lo Stato islamico. AGC Communication 2014

Cannavicci M. Psyops. Le operazioni psicologiche nei conflitti asimmetrici. Giornale di medicina militare 2005.

De Rosa Corrado. Nella mente di un jihadista. Per una psicologia dell'Isis. Corriere della Sera ebook 2016

D'Auria S. Il terrorismo islamista dalle origini allo stato islamico. Strategie e normative di contrasto. Rassegna penitenziaria e criminologica febbraio 2015

Mastronardi V. Manuale per operatori criminologici e psicopatologi forensi. Giuffrè editore 2012

Mastronardi V. Moral retreat mechanism in the formation of the terrorist and Islamic terrorism. Università degli Studi di Roma La Sapienza facoltà di Medicina. Department of psychiatry and psycological medicine. Teaching of forensic psychopathology. Observatory of behaviors and deviance.

Oasis numero 23. Il Corano e i suoi custodi". Marsilio Editori 22 giugno 2016.

Palermo G., Mastronardi V. Personalità e psicopatologie. Il profilo criminologico dalla scena del crimine ai profili socio-psicologici. Giuffrè editore 2005.

\section{Analisi del fenomeno terrorismo internazionale Analysis of the international terrorism phenomenon \\ Maria Ligorio}

Avvocato. Criminologo. In collaborazione con il Centro Ricerca sulla Sicurezza e Terrorismo, Italy

II terrorismo internazionale negli ultimi anni ha subito cambiamenti sostanziali che ne hanno ridefinito strategie e spazi d'azione. L'inizio della transazione verso un nuovo modello di terrorismo è stato segnato dai tragici attentati dell'11 settembre del 2011 che ha cambiato la percezione del pericolo anche agli occhi dell'opinione pubblica. La comunità internazionale ha preso coscienza che non solo la conoscenza del fenomeno è precognizione essenziale per improntare risposte di prevenzione, sicurezza e legislative così come, peraltro, è accaduto per la legislazione antimafia, benchè si trattasse di contrastare un fenomeno radicato nella società e non fosse una emergenza, ma ha preso coscienza di un problema che richiede un agire congiunto ed uno sforzo comune costanti. Con gli anni il fenomeno ha assunto caratteristiche diverse, utilizzando strumenti e tecnologie sempre più avanzate ma ha mantenuto intatti i connotati tradizionali: l'estrema violenza distruttrice di vittime innocenti e l'eclatante necessità di una platea. Nel pieno rispetto del travaglio insito in tutte le definizioni del terrorismo formulate nel corso del tempo é dunque difficile tracciare i lineamenti di un fenomeno poliedrico, liquido e mutevole appare invece opportuno non definirlo bensì descriverlo cioè soffermarsi su una "descrizione funzionale" del terrorismo basata esclusivamente su osservazioni e considerazioni di natura empirica. L'Unione Europea (UE) non formula una definizione del terrorismo, ma si sofferma sulla nozione di reati terroristici, utilizzando appunto il plurale, e ne elenca numerose fattispecie. Per la UE "sono reati terroristici: gli atti intenzionali [...] che, per la loro natura o contesto, possono arrecare grave danno a un Paese 0 a un'organizzazione internazionale, quando sono commessi al fine di intimidire gravemente la popolazione o costringere indebitamente i poteri pubblici o un'organizzazione internazionale a compiere 0 astenersi dal compiere un qualsiasi atto, o destabilizzare gravemente le strutture politiche fondamentali, costituzionali, economiche o sociali di un Paese o di un'organizzazione internazionale." Oltre ai reati terroristici, la UE annovera i reati riconducibili a un'organizzazione terroristica, i reati connessi alle attività terroristiche, specifica cosa deve intendersi per organizzazione terroristica, prevede la punibilità tanto della direzione di tale organizzazione quanto la partecipazione alle attività di tale organizzazione, anche nel caso vengano fornite informazioni o erogati mezzi materiali, ovvero tramite qualsiasi forma di finanziamento delle sue attività nella consapevolezza che detta partecipazione contribuirà alle attività criminose dell'organizzazione. Lo sviluppo di una legislazione di contrasto del terrorismo, quindi, si può dividere in due fasi principali: quella precedente ai devastanti attentati dell'11 settembre 2001 e quella successiva, caratterizzata da misure di carattere innovativo adottate a ritmo serrato a fronte di un'esigenza ritenuta d'emergenza e come tale prioritaria. Per quanto riguarda I'Italia, essendo un Paese con una importante esperienza in materia di minaccia terroristica riconducibile a molteplici matrici, nonché di notevoli successi nel settore del contrasto, il legislatore ha da tempo previsto il delitto di attentato per finalità terroristiche 0 di eversione (art. 280 c.p.), ma non solo, in quanto accanto al reato di associazione sovversiva (art. 270 c.p.), il legislatore ha collocato il reato di associazione con finalità di terrorismo o di eversione dell'ordine democratico, inteso a perseguire le condotte sopra descritte ogni qual volta fossero riferibili ad associazioni intenzionate a compiere atti di violenza per scopi di eversione dell'ordine costituzionale. Successivamente ai tragici eventi dell'11 settembre 2001, il legislatore italiano ha stabilito che la finalità di terrorismo ricorre altresì quando gli atti di violenza sono rivolti contro uno stato estero, un'istituzione e un organismo internazionale, prevedendo così il reato di terrorismo internazionale (art. 270 bis c.p.). A brevissima distanza dalla riforma d.I. 18 febbraio 2015, n.7 conv. con modifica dalla I. 17 aprile 2015 n. 43, il legislatore italiano è nuovamente intervenuto sulle norme di contrasto al terrorismo mediante la legge del 28 luglio 2016 n. 153 con la quale adegua il nostro ordinamento ad una serie di impegni internazionali in materia di terrorismo, apportando tra l'altro significative modifiche al codice penale. In Particolare, alla già nutrita costellazione di norme vengono apportate nuove fattispecie incriminatrici quali i delitti di finanziamento di condotte con finalità di terrorismo (art. 270 quinquies1 c.p.), sottrazione di beni 0 denaro sottoposti a sequestro (art. 270quinquies 2 c.p.) atti di terrorismo nucleare (art. 280-ter c.p.), prevedendo altresi una nuova ipotesi di confisca obbligatoria, diretta e per equivalente, per tutti i reati commessi con finalità di terrorismo (art. 270-septies c.p.). Con la legge 28 luglio 2016 n. 153 autorizza la ratifica e dà piena ed intera esecuzione a diverse convenzioni internazionali. Per ultimo, la nuova disciplina in materia di prevenzione del riciclaggio e di finanziamento del terrorismo del 25 maggio 2017 n. 90 di attuazione della Direttiva UE 849/2015 (Quarta Direttiva), a cui è sopraggiunta una proposta di Direttiva della Commissione Europea che modifica la Quarta Direttiva con lo scopo di rafforzare ulteriormente l'azione di contrasto e prevenzione del riciclaggio del finanziamento al terrorismo. Da questa prospettiva, si é piu' volte sottolineato l'importanza che gli atti di preparazione rivestono nella questione in oggetto e come nella pratica giudiziaria gli Stati hanno ricorso sempre piu' frequentemente a capi d'imputazione per fatti che precedono la commissione degli atti di terrorismo. Esempi concreti li possiamo riscontrare nella cronaca di tutti i giorni nel numero di espulsioni effettuati a ritmi molto alti come prevenzione antiterrorismo, con cui gli investigatori cercano e puntano chiunque si dimostri potenzialmente pericoloso anche se non ha neanche tentato di commettere un atto di terrorismo. Questo breve excursus è stato doveroso per comprendere come il legislatore ha gestito in passato l'emergenza terrorismo e quali strumenti utilizza oggi per l'omonimo fenomeno internazionale. In sintesi la disciplina della prevenzione del terrorismo internazionale si basa: sulla lotta al finanziamento del terrorismo; sul contrasto all'accesso ad armi convenzionali e di distruzione di massa; sui controlli al movimento di individui sospetti terroristi.

\section{Bibliografia}

Betti S. (2015), Le armi del diritto contro il terrorismo, Milano, FrancoAngeli. Razzante R. (2011), Finanziamento del terrorismo e antiriciclaggio, Nuova Giuridica. 
Pisano V. (1998), Introduzione al Terrorismo Contemporaneo, Roma, Sallustiana, e dello stesso autore, Conflitti Non Convenzionali nel Mondo Contemporaneo, (2002), Roma, Edizioni Rivista Marittima.

Centonze S., Giovedì L. (2016), Terrorismo e legislazione d'emergenza, Key editore. Mugavero R., Razzante R. (2016), Terrorismo e nuove tecnologie, Pacini Giuridica. Presidenza del Consiglio dei Ministri, Relazione sulla Plitica Informativa e della Sicurezza, Pubblicazione semestrale.

Decisione Quadro del Coniglio dell'Unione Europea del 13 giugno 2002 Sulla Lotta Contro il Terrorismo (2002/475/GAI)

\section{La coppia dell'acido: un patto perverso tra immaturità e dipendenza. Uno studio grafologico The acid-attack couple: A perverse pact between immaturity and addiction. A graphological study}

Elena Manetti

Grafologa, Presidente Arigraf Nazionale (Associazione Ricerca Grafologica), Referente Lazio AGP (Associazione Grafologi Professionisti) e Membro del Comitato Tecnico Scientifico dell'AGI (Associazione Grafologica Italiana); Direttore responsabile della rivista "Stilus, percorsi di comunicazione scritta"; Docente al corso di "Grafologia Forense e Aspetti clinico valutativi", Dipartimento S.A.I.M.L.A.L. Sezione di Medicina Legale, Università degli Studi Sapienza di Roma, Italy

I criminologi sostengono che quando due persone si uniscono in coppia per commettere un atto deviante, le componenti singole dei due partecipanti vengono amplificate all'ennesima potenza fino ad arrivare ad una vera e propria "follia a due". E' il caso della coppia Alex Boettcher e Martina Levato, lui il vero "architetto" del folle piano, sadico e perverso, con una forte capacità manipolatoria, lei in stato di totale soggezione, completamente priva di autostima, passiva e vulnerabile. In un crescendo di comportamenti sempre più trasgressivi anche e soprattutto sessuali, con la donna ridotta a un'umiliante schiavitù, marchiata a fuoco e in un totale isolamento sociale, i due concepiscono un "piano criminale" che, secondo le ricostruzioni in sede giudiziaria, era quello di aggredire ragazzi con cui la Levato aveva avuto in passato relazioni sentimentali allo scopo di "purificarla" e renderla "degna" di essere la compagna del Boettcher. Gli agguati sono stati 4, tra maggio e dicembre del 2014 , con la Levato che agisce o con il coltello (tenta di evirare un suo ex fidanzato) o gettando acido addosso ai malcapitati, e il Boettcher che assiste 0 interviene per bloccare 0 inseguire la vittima. La Levato è stata condannata con rito abbreviato in primo grado a 20 anni di carcere e il Boettcher a 34, anche se si continua a professare innocente. A entrambi è stato tolto il figlio che è nato in carcere e che è stato dichiarato in stato di adottabilità. Non è facile capire come si possa arrivare a tale punto di follia e sicuramente gli esami psichiatrici e psicologici chiariranno la struttura di personalità che ha indotto i due protagonisti agli atti devianti. La mia relazione si basa sull'osservazione e l'analisi della loro scrittura come spontanea espressione del loro comportamento. Come è ovvio, la scrittura è un prodotto del cervello, meno chiare sono le correlazioni tra la scrittura e le attività neuronali responsabili, da cui derivano i comportamenti. Particolarmente interessanti sono gli studi, che si sono sviluppati negli ultimi dieci anni in varie parti del mondo, sui sistemi emozionali ed i rapporti con la personalizzazione comportamentale. Le scoperte delle neuroscienze hanno sottolineato la grande importanza delle emozioni, stati mentali delle cui motivazioni possiamo non essere del tutto consapevoli, ma che guidano nelle decisioni più importanti e strutturano in un certo tipo di personalità e tutti quelli che si occupano di scrittura sono ben consapevoli di quanto lo stato emotivo possa riflettersi sulla scrittura stessa. Tutti gli individui reagiscono agli stimoli emozionali secondo una loro propria modalità che si presenta costante e che in un certo modo "programma" il loro comportamento e il loro modo di affrontare le relazioni con gli altri e le situazioni ambientali. La grafologia, in interazione con la psicologia, può in effetti dare un approfondimento nell'analisi di una personalità, perché la scrittura coinvolge, nel suo svolgersi, diverse capacità, non solo di astrazione, di coordinamento motorio, di corretta ed esatta capacità di modulare i corrispondenti gruppi muscolari, di capacità associativa, di percezione visiva integrale, di organizzazione e orientamento spaziale, ma anche di capacità espressiva, di controllo, di capacità comunicativa o di nascondimento. La scrittura infine riproduce il grado di adattamento o di disadattamento di chi scrive e può dare un utile contributo nell'indagare le motivazioni più profonde di un possibile atto criminale.

\section{Bibliografia}

Avé Lallemant U. Segnali d'allarme. Grafologia e diagnosi psichica della personalità, Roma, Armando ed, 1997.
Bastin C., de Castilla D. Graphologie, le psychisme et ses troubles, Paris, Ed. Laffont, 1990.

Blanquefort d'Anglars M. Motivazioni e Compensazioni, approccio grafologico e psicologico, Manduria (TA) Giordano editore, 2010.

Boille N. Il gesto grafico gesto creativo, Roma, Borla, seconda ed. 2007.

Boille N., Manetti E. La nostra scrittura, un test per riconoscersi, Sovera Roma, Edizioni, 2010.

Callieri B. I comportamenti indotti. Considerazioni di uno psicopatologo, in “Criminologia, Psichiatria Forense e Psicologia Giudiziaria. Delfino, Roma, 1996.

Coda S. Coppie criminali. Amarsi, vivere, uccidere. Centro Scientifico Editore, Torino, 2001.

Colo C., Pinon J. Traité de Graphologie dynamique de l'écriture, Parigi, Expansion scientifique française, 2002.

De Cataldo Neuburger L. La donna serial killer, in "La criminalità femminile tra stereotipi e malintese realtà", Cedam, Padova, 1996.

Lefebure F., Van den Broek d'Obrenan C. Le trait en graphologie: indice constitutionnel, Masson, Paris, 1986

Fornari U. Trattato di Psichiatria Forense, Utet, Torino, 1997.

Gille Maisani J.Ch. Psicologia della scrittura, Napoli, Liguori, 1990.

Mastronardi V. Il plagio tra persistenza e negazione, in “Criminologia, Psichiatria Frense e Psicologia Giudiziaria, Roma, 1996.

Mastronardi V., Bidoli S., Calderaro M. Grafologia Giudiziaria e Psicopatologia Forense, Giuffré Editore, Milano, 2010.

Moretti G. Scompensi, anomalie della psiche e grafologia, Edizioni Messaggero Padova, Padova, 2000.

Muller W.H., Enskat A. Diagnostica grafologica. Principi, possibilità e limiti. Padova, II Messaggero, 1995.

Zucchi I. Grafologia della devianza, CTS, Città di Castello, 1998.

Witkowski F. Psicopatologia e scrittura, Epsylon Editrice, Roma, 2012.

\section{Stalking nei confronti di operatori di contesti socio-sanitari: risultati preliminari di una ricerca nella regione Sardegna Stalking of social healthcare workers: Preliminary results of a study conducted in Sardinia, Italy}

Francesca Manunza, ${ }^{1}$ Vincenzo Mastronardi, ${ }^{2}$ Monica Calderaro ${ }^{3}$

${ }^{1}$ Dirigente Medico Psichiatra ATS Sardegna, Psicoterapeuta, Criminologa, Psicopatologa Forense; ${ }^{2}$ Già Direttore della Cattedra di Psicopatologia Forense, $1^{\text {a }}$ Facoltà di Medicina, Dipartimento di Neurologia e Psichiatria, Sapienza Università Roma, Direttore del Master in Criminologia, Unint Università degli Studi Internazionali di Roma e Università Telematica degli Studi di Roma Unitelma Sapienza, Insegnamento di Criminologia cdl SCAMS, Università Telematica degli Studi di Roma Unitelma Sapienza; ${ }^{3}$ Criminologa Ph.D. Internazionale, Dottore in Psicologia ad indirizzo Clinico, Grafologa, Perito Grafologo, Docente e Responsabile didattico nel Corso di Formazione in Grafologia forense "Sapienza" Università di Roma, Docente Master in Criminologia UNINT Università degli Studi Internazionali di Roma, Ricercatore Istituto Internazionale di Scienze Criminologiche e Psicopatologico Forensi (MIUR codice car n. 62065CSS), Tutor Area disciplinare Criminologica Università degli Studi di Roma Unitelma Sapienza, Italy

Diversi studi hanno posto in evidenza come per particolari categorie sociali e professionali esista un maggior rischio di essere vittima di stalking. Le relazioni professionali possono costituire un terreno favorevole su cui si possono originare fraintendimenti all'origine di comportamenti di stalking, esse prevedono un determinato setting all'interno del quale l'interazione tra individui è legittimata e preservata, vi è tuttavia un certo grado d'intimità insito nella natura della relazione stessa che può essere oggetto di fraintendimento da parte dell'assistito (Curci P. et al. 2003). I medici e i professionisti della salute in genere, sono a rischio di subire molestie durante l'esercizio della loro professione, in particolare da parte dei loro pazienti; tale rischio si è evidenziato maggiore per psichiatri e psicoterapeuti (Praus P. et al. 2017). Secondo Mclvor e colleghi, il (21\%) degli psichiatri è stato vittima di stalking, la durata dei comportamenti di stalking sugli operatori della Salute Mentale va da qualche settimana a diversi anni (Ostermeyer et al. 2016 da Mc Ivor et al. 2008).

Scopo. II lavoro si propone di ottenere una maggiore comprensione e rappresentazione della frequenza con cui le Molestie Assillanti si verificano nei confronti di operatori sociosanitari di strutture pubbliche in Sardegna.

Materiali e Metodi. E' stato somministrato un questionario anonimo, preparato ad hoc, dopo una rassegna della letteratura, ad un campione di (265) operatori della Salute Mentale della Sardegna: di questi hanno risposto (210), di essi abbiamo considerato (187) operatori, (esclusi 23 operatori: riabilitatori psichiatrici, educatori professionali, assistenti sociali) e scelto di studiare la vittimizzazione subita da medici psichiatri, psicologi, infermieri, operatori sociosanitari. 
Risultati. Nel nostro campione i soggetti che hanno segnalato molestie assimilabili al reato di stalking sono rappresentati da $n=30$ operatori su $\mathrm{n}=187$ del campione totale (16\%). II sesso femminile risulta maggiormente rappresentato con $\mathrm{F}=23 / 30$ (77\%) rispetto a quello maschile $\mathrm{M}$ $7 / 30$ (23\%). II rischio di essere vittima di molestie è risultato maggiore per il gruppo di psichiatri e psicologi rispetto agli infermieri. Tra le vittime $n .=18$ erano medici psichiatri $(60 \%), n .=3$ psicologi $(10 \%), n .=9$ infermieri (30\%). L'età media delle vittime è di 51 aa per i maschi e 40 aa per le donne. Le vittime in $n .=11 / 30$ casi $(37 \%)$ erano celibi/nubili, in $n .=4 / 30(13 \%)$ divorziate, in $n .=15 / 30(50 \%)$ (sposate/conviventi); il titolo di studio è medio alto, essendo uno studio in ambito sanitario. Gli Stalker sono in $\mathrm{n} .=23$ casi $(77 \%)$ un paziente 0 ex-paziente seguito dal professionista, in $n .=4$ casi $(14 \%)$ un familiare del paziente (marito/fratello), in $\mathrm{n} .=3$ casi (10\%) la tipologia dello stalker non è specificata. Le diagnosi psichiatriche prevalenti negli stalker (M) sono state in $n .=9$ stalker Disturbo da Uso di Sostanze, in $n .=9$ stalker Psicosi (30\%), in $\mathrm{n} .=9$ stalker Disturbo di Personalità $(30 \%)$, in $n .=2$ casi $(7 \%)$ Disturbo dell'Umore, $n .=8$ molestatori avevano una Doppia Diagnosi (27\%). Nelle donne $n .=3$ stalker $(10 \%)$ avevano una diagnosi di Disturbo dell'Umore, $n .=3$ stalker $(10 \%)$, avevano Diagnosi di Disturbo di Personalità, $n .=4$ stalker donne avevano un Disturbo da Uso di Sostanze (13\%), n.=4 stalker di sesso femminile hanno una Doppia Diagnosi (13\%). Sebbene il caso più comune sia quello di un molestatore che perseguita un operatore di sesso opposto $(77 \%)$ dei casi, nel $(23 \%)$ la diade molestatore-molestato è dello stesso sesso. La richiesta di aiuto ai colleghi è stata utilizzata in $n .=10$ casi $(33,3 \%)$, la segnalazione ai superiori è stata effettuata in n. $=10$ casi dal $(33,3 \%)$ delle vittime, la richiesta di aiuto ai familiari del paziente è stata effettuata in $n .=9(30 \%)$, delle vittime, la richiesta di aiuto ai familiari propri in $n=2$ casi, $(7 \%)$, la denuncia ai carabinieri in $n .=4$ casi, $(13 \%)$ delle vittime, in $n .=2$ casi $(7 \%)$ è stata effettuata la segnalazione ai carabinieri senza denuncia, in $n .=2$ casi (7\%) è stato necessario cambiare posto di lavoro.

Discussione. I professionisti della salute mentale sono ad elevato rischio di vittimizzazione. Lo strumento principale a disposizione dei servizi è l'approccio d'èquipe ai casi multiproblematici evitando che la diade operatore designato e paziente s'isoli (Curci et al. 2003, Sindrome delle Molestie Assillanti). La sensazione d'impotenza ed isolamento che frequentemente è presente nelle vittime di stalking, anche nel nostro campione, viene evidenziata da diversi studi in cui il (10\%) delle vittime intervistate dichiara di aver pensato al suicidio come via d'uscita (Purcell et al. 2005).

\section{Bibliografia}

Galeazzi G.M., Curci P., Secchi C. (2003), La sindrome delle molestie assillanti (stalking), Manuali di Psicologia, Psichiatria, Psicoterapia, Bollati Boringhieri.

Ostermeyer B. et al. (2016), da Mclvor R. J., Potter L., Davies L., (2008) Stalking behaviour by patients towards psychiatrists in a large mental health organization, Int J Soc Psychiatry; 54(4):350-7 in Stalking and Violence, Psychiatr Clin North Am. 39(4): 663-673.

Praus P., Riedel-Heller S., Dressing H. (2017), Stalking of psychiatrists and psychotherapists: result of an online survey, Der Nervenarzt.

Purcell R., Powell M.B. and Mullen P.E. (2005), Clients who stalk psychologists: prevalence, methods, and motives. Professional Psychology: Research and Practice 36: 537-543, citato in McEwan T.E. e Strand S. (2013).

\section{Stalking, malattia mentale, imputabilità: riflessioni su 20 casi Stalking, mental disease, imputability: Thoughts on 20 cases}

Francesca Manunza, ${ }^{1}$ Giampaolo Pintor ${ }^{2}$

${ }^{1}$ Dirigente Medico Psichiatra ATS Sardegna, Psicoterapeuta, Criminologa, Psicopatologa Forense; ${ }^{2}$ Medico Psichiatra, Socio fondatore e Membro del Consiglio Direttivo della Società Italiana di Psichiatria Forense, Italy

Lo stalking è il prodotto di un'interazione tra contesto (compresa la relazione diadica tra stalker e vittima) e fattori individuali come la psicopatologia (McEwan T. et al. E. 2013). Coloro che assumono comportamenti di stalking sono spesso affetti da disturbi mentali che possono avere un ruolo diretto o indiretto nel perpretare il comportamento. In letteratura si rileva che gli stalker d'ex-partner rappresentino la sottocategoria più ampia di stalker; Spitzberg riporta una percentuale media del (49\%) di stalker d'ex-partner su 40 studi (Sheridan et al. 2003 da Spitzberg 2002), essi mostrano una maggiore diversità di comportamenti di stalking con un tasso di logoramento più veloce. Vari studi indicano una maggior prevalenza di psicosi tra coloro che perseguitano estranei o conoscenti rispetto agli stalker d'ex partner (25\%/11\%) (McEwan T. et al. E. 2013 da Mohandie 2006). Alcuni studi, oltre il pre- sente, indicano una prevalenza alta di Disturbi di Personalità Nas negli stalker, suggerendo che il comportamento di stalking possa essere associato ad una combinazione di tratti di personalità di diversi disturbi. (McEwan T. et al. E. 2013, Rosenfeld 2003).

Scopo. II presente contributo si propone di trovare dei riscontri empirici ai dati suggeriti dalla letteratura scientifica di riferimento.

Materiali e Metodi. Abbiamo esaminato il fascicolo peritale di 20 stalker sottoposti a valutazione psichiatrico-forense per il reato di stalking (art. 612/bis) negli anni 2016/2017, dal Servizio di Psichiatria Forense di Cagliari. Tramite un'apposita griglia, che ha permesso di garantire l'anonimato e la non riconoscibilità degli autori di reato, sono state analizzate alcune variabili demografiche (età, sesso, stato civile, scolarità), le diagnosi degli stalker, la tipologia della vittima oggetto di stalking, le forme di stalking esercitate, con particolare attenzione alle manifestazioni di violenza, la durata dello stalking e alcuni dati di tipo psichiatrico-forense.

Risultati. II (95\%) degli stalker esaminati è di sesso maschile $n .=19$ (M) (95\%), non ha una relazione affettiva stabile, ha avuto almeno un accesso al Dipartimento di Salute Mentale, il (70\%) degli stalker è disoccupato o pensionato. Dal punto di vista psichiatrico forense $n .=14$ stalker $(70 \%)$ sono affetti da vizio parziale di mente, $n .=5(25 \%)$ da vizio totale di mente, $n .=1$ stalker è stato riconosciuto capace d'intendere e volere; $n .=13$ erano pericolosi socialmente. II (45\%) degli stalker sono affetti da Disturbo di Personalità Nas. Le diagnosi prevalenti (Asse I) sono: D. da Addiction in n. $=8$ stalker $(40 \%)$, Disturbi Bipolari e disturbi correlati $n .=6$ stalker (30\%), Disturbi dello Spettro della Schizofrenia e altri disturbi Psicotici, in n. $=5$ casi $(25 \%)$. II (45\%) delle vittime di stalking sono ex-partner.

Discussione. Vista l'alta possibilità di comportamenti violenti nello stalking, presenti nella metà dei casi di stalking di ex-partner, e l'alta possibilità di reiterazione del reato in particolare tra gli stalker affetti da Disturbo di Personalità, il Servizio di Psichiatria Forense di Cagliari in accordo con gli Uffici Giudiziari, sta lavorando a delle linee guida che prevedono la creazione di centri che si occupino della cura dello stalker al fine di contenere la recidiva di reato.

\section{Bibliografia}

McEwan T.E. e Strand S. (2013), The role of psychopathology in stalking by adult strangers and acquaintances, Australian \& New Zealand Journal of Psychiatry 47(6) 546-555.

McEwan T.E. e Strand S. (2013), da Mohandie K., Meloy J.R., McGowan M.G., et al. (2006) The RECON typology of stalking: Reliability and validity based on a large sample of North American stalkers. Journal of Forensic Sciences 51: 147-155.

Rosenfeld B. (2003), Recidivism in stalking and obsessional harassment. Law and Human Behavior 27: 251-265.

Sheridan, L. P., Blaauw E., Davies G. (2003), da Blaauw, E., \&Winkel, F.W. (2002), Stopping stalking, Manuscript submitted for publication, in Stalking: Knowns and Unknowns, Trauma, Violence, \& Abuse, Vol. 4, No. 2, April 2003 148-162, in Stalking: Knowns and Unknowns, Trauma, Violence, \& Abuse, Vol. 4, No. 2, April 2003 148-162.

Sheridan, L. P., Blaauw E., Davies G. (2003), da Spitzberg B. H., (2002), The tactical topography of stalking victimization and management. Trauma, Violence \& Abuse, 3, 261-2 in Stalking: Knowns and Unknowns, Trauma, Violence, \& Abuse, Vol. 4, No. 2, April 2003 148-162.

\section{Caravaggio genio e criminale: uno studio grafologico Caravaggio as a genius and a criminal: A graphological research} Elena Marchetti

Grafologa, Esperta in analisi e comparazione delle grafie, Criminologa; Docente di Grafologia, "Sapienza" Università di Roma, Consulente e perito in ambito giudiziario; Coach aziendale e responsabile settore grafologia Accapierre S.r.l. Milano. Licensed Master Practitioner of Neuro-Linguistic Programming, certificato Society N.L.P., Italy

Michelangelo Merisi detto il Caravaggio: il matrimonio tra cielo e inferno. Artista sublime e, allo stesso tempo, uomo violento e delinquente. Una carriera criminale che va dal possesso illegale di armi, alla diffamazione, al gioco d'azzardo, alla rissa, alle lesioni personali, fino all'omicidio per cui, nel 1606, viene condannato a morte dal Tribunale dello Stato della Chiesa. La personalità di Caravaggio (caratterizzata, come la sua pittura, da contrasti di luci e ombre) insieme alla sua storia di vita (da cui emergono tendenze distruttive e autodistruttive) permettono oggi di ipotizzare che il pittore soffrisse di un disturbo di personalità che lo portava a distruggere sistematicamente i legami personali, ma che non compromise il suo genio pittorico, che anzi si nutriva di queste tinte fosche e tenebrose. Lo studio grafologico presentato non ha lo scopo 
di parlare del virtuosismo tecnico pittorico, ma dei chiaroscuri, delle "ombre", tanto rilevanti nel pensiero di Carl Gustav Jung e di Christopher Bollas, che ci dicono quanto nella vita di Caravaggio ci sia stata molta più oscurità che luce. La notte, come sappiamo, è piena di vita nascosta e spesso vi accadono le cose più tremende. L'oscurità è la terra dell'inconscio e delle pulsioni più inconfessabili. L'esame della scrittura di Caravaggio fa luce proprio sugli aspetti più inquieti della sua personalità che lo hanno portato alla perversione e all'omicidio. L'analisi grafologica permette uno studio che evidenzia: la sua profonda inquietudine; la presenza di un disturbo del controllo degli impulsi; un potente conflitto edipico con profonda angoscia di castrazione.

\section{Bibliografia}

Bollas C. L'ombra dell'oggetto. Psicoanalisi del conosciuto non pensato, Borla ed., Milano 1989.

Conklin J.E. Criminology, quarta edizione, Macmillan, New York 1992.

De Zulueta F. Dal dolore alla violenza. Le origini traumatiche dell'aggressività, Raffaello Cortina Ed., Milano 1999.

DSM-5, Manuale Diagnostico e Statistico dei Disturbi Psichiatrici (Edizione italiana a cura di M. Biondi), Milano, Ed. Raffaello Cortina (prima ed.), Milano 2014

Hazelwood R. Ossessioni Criminali, Ed. Mediterranee, Roma 2006.

Holmes R.M., Holmes S.T. Profiling Violent Crimes: An Investigative Tool, $3^{\mathrm{a}}$ ed. Thousand Oaks, CA, London, New Delhi: Sage 2002.

Jung C.G. Lotta con l'Ombra (1946) in Opere X Tomo II, Bollati Boringhieri, Torino 1986; Lewis CN. Caravaggio's Imagery of Death and Allusion Ed. American Imago, Otterbein 1986.

Mastronardi V. Bidoli S.A., Calderaro M. Grafologia Giudiziaria e Psicopatologia forense, Giuffrè Ed. Milano $2^{\mathrm{a}}$ ed. 2010.

Moretti G. Trattato scientifico di perizie grafiche su base grafologica Messaggero Ed. Padova ed. 2002

Peugeot J., Lombard A., De Noblens M. Manuale di grafologia Masson Ed., Milano 1992. Pulver M. La simbologia della scrittura Boringhieri, Torino 1983.

\section{Rivediamo il concetto di vittimologia Let's review the concept of victimology}

\section{Giovanni Neri}

Patrocinante innanzi alla Corte di Cassazione ed alle Magistrature Superiori, Italy; Full Professor of Law at Miami International University, USA; Direttore della Collana "Jus \& Comparative Law", Collana di Diritto e Diritto Comparato, Sapienza, Università di Roma, Italy

La criminologia si è per decenni occupata unicamente dei reati e dei loro autori, disinteressandosi della vittima e delle implicazioni da essa rivestite nella commission e nello sviluppo dei fatti delittuosi. È solo a partire dagli anni '40 del XX secolo che nasce e si sviluppa la vittimologia, ossia la scienza che studia la vittima e la sua personalità, i "suoi tratti biologici, psicologici e morali, le sue caratteristiche socio-culturali, le sue relazioni con l'autore del reato ed infine il suo ruolo e l'eventuale influenza nella genesi e nella dinamica del delitto". II termine è stato coniato da un avvocato israeliano, Mendelson, per indicare dunque una branca della criminologia che esamina la vittima da più angolazioni prospettiche e, mediante l'esame congiunto della diade vittima-autore, da un lato consente una migliore comprensione della criminogenesi e criminodinamica dei fatti e dei rischi di vittimizzazione e, dall'altro, permette l'elaborazione di strategie preventive e la progettazione di programmi e strumenti di reinserimento sociale e ristoro delle vittime e dei familiari. Si tratta pertanto di una scienza multifunzionale con scopi diagnostico-preventivi e di tipo riparativo, che prende ad oggetto la vittima in relazione al rapporto psicologico sociale con il reo e in funzione del suo eventuale apporto nella dinamica criminogenetica. La disciplina, sistematica e di tipo empirico, deve il suo sviluppo e la sua autonomia scientifica principalmente agli studi di Von Henting, Mendelson ed Ellenberg. I primi elaborarono i fondamentali concetti di criminale-vittima - sottolineando come non si nasca criminali osuccubi, ma si acquisisca un ruolo piuttosto che l'altro a seconda degli eventi - e di vittima potenziale - individuando la predisposizione fisica 0 psicologica di alcuni soggetti a divenire bersagli preferenziali di reato. Mendelson poi, valorizzando la necessaria interrelazione tra vittima e autore nell'economia dei delitti, classificò le prime in relazione al grado di colpa ad esse attribuibile nella dinamica criminale.Il lavoro quindi tende a rivisitare il concetto di Vittimologia sia nella classificazione delle vittime , sia in relazione alle predisposizioni vittimogene, sia in relazione alle nuove tipologie di Autore e sia in relazione alla Vittima, nel contesto del processo penale italiano.

La classificazione delle vittime e le cd. predisposizioni vittimogene. Come sin ora esposto, dunque, la vittimologia consente di meglio chiarire l'effettiva portata degli apporti vittimogeni alla realizzazione del fatto di reato e permette di approfondire il processo di vittimizzazione, la rilevanza e la natura del danno psicologico esistenziale patito in conseguenza di esso. Per vittima si intende colui che ha patito un pregiudizio, morale o patrimoniale, giuridicamente e socialmente riconosciuto da parte della collettività, per effetto dell'azione criminale, o per utilizzare la dicitura della Decisione Quadro del Consiglio dell'Unione Europea, "la persona fisica che ha subito un pregiudizio, anche fisico o mentale, sofferenze psichiche, danni materiali causati direttamente da atti o omissioni che costituiscono una violazione del diritto penale". I criminologi hanno nel tempo elaborato svariate distinzioni, per identificare le diverse tipologie di vittime, in chiave didattico-classificatoria. Una prima ripartizione distingue le cd. vittime reali da quelle simulatrici ed immaginarie. Queste ultime in particolare, per le ragioni più disparate, sostengono falsamente di essere oggetto di comportamenti criminosi, con dolo (vittima simulatrice) 0 per un'errata percezione della realtà riconducibile per lo più a reazioni psicopatologiche di varia natura (vittima immaginaria). Ancora, tra le vittime reali si distinguono quelle attive e passive. Le prime divengono tali in ragione della specifica professione espletata (ad esempio, le forze dell'ordine che, per sventare una rapina, rimangono colpiti in un conflitto a fuoco) o comunque partecipano, per diverse vie, alla realizzazione del fatto. Si individuano a questo proposito vittime favorenti e consenzienti - che agevolano o autorizzano la commissione del delitto - provocatrici - che subiscono violenza per aver provocato la reazione delinquenziale con precedenti comportamenti di tipo aggressivo - o disonoranti - che determinano la condotta reattiva del congiunto di chi sia offeso nell'onore sessuale. Le seconde, invece, vengono coinvolte nella genesi del reato in modo del tutto accidentale ed indiscriminato (si pensi a chi rimanga leso da attività minatorie di tipo terroristico). Tra queste ultime rientrano anche le cd. vittime simboliche, scelte per colpire in modo esemplare e punitivo un determinato gruppo o compagine sociale. Da ultimo, si individuano vittime trasversali - ossia familiari colpiti in particolare da forme di vendetta di natura criminale - collettive - indiscriminatamente lese dall'abuso dei poteri tradizionali, in ambito statuale, sociale o familiare - e preferenziali, individuate e scelte per la specifica posizione economica ricoperta oper la sussistenza, comunque, di circostanze di agevolazione del delitto. A quest'ultimo proposito, gli studiosi di psicanalisi hanno elaborato la teoria della subcosciente tendenza a soffrire, come forma di manifestazione di una più generale inclinazione verso forme autodistruttive della personalità, individuando categorie di soggetti che, perragioni di natura fisica, sociale o psicologica, si presentano maggiormente inclini a subire comportamenti di tipo criminale. Si parla in tal caso di predisposizioni vittimogene, ove gli elementi che predispongono ad assumere il ruolo di vittima devono porsi in rapporto di causalità diretta con il fatto commesso e la qualità della stessa esplica una funzione fondamentale nellarealizzazione, 0 addirittura nella stessa ideazione del crimine compiuto. Le predisposizioni possono essere di natura biofosiologica, sociale o psicologica, e possono avere genesi innata o acquisita. Nel primo caso, appaiono tipizzate da particolari componenti fisiche o biologiche che accompagnano l'individuo geneticamente e fisiologicamente (l'età, il sesso o vizi di mente); nel secondo, sono il frutto di connotazioni derivate da fattori acquisitivi di tipo sociale opsicologico (status o infermità non congenite). Da ultimo, le predisposizioni possono essere temporanee, se circoscritte a determinati e finiti archi temporali, o permanenti, e possono avere o meno natura conscia.

Vittima e autore del reato. L'interesse criminologico per la vittima è strettamente connesso allo studio dell'autore del reato, nel tentativo di individuare "la criminogenesi e il passaggio all'azione nella loro verità sostanziale e dinamica", oltre che le reali logiche per le quali un dato soggetto può entrare a far parte passivamente di determinati circuiti criminosi. Inoltre, l'analisi dei meccanismi d'incontro tra i due soggett del reato consente di stabilire il grado e l'estensione della pericolosità criminaledel reo, valutando l'eventuale legameintersubiettivo che lo muove a un dato fattoovvero, al contrario, un'inclinazione costante eindiscriminata a delinquere. Da ultimo, permette di sensibilizzare le vittime a modulare i comportamenti tenuti con finalità di tipo preventivo. In altre parole, non potendosi a priori controllare la proliferazione del crimine, si tenta di arginarne gli effetti veicolando o comunque tentando di controllare i comportamenti vittimogeni. È comunque indubbio che determinate tipologie di realtà gruppali, da cui derivanoevidenti vincoli di interdipendenza, rendono più semplice e frequente la perpetrazione di condotte delinquenziali. Si pensi alle cd. bande criminali giovanili o più in generale al raggruppamento familiare, particolarmente significativo nella scelta della tipologia del delitto e della relativa vit- 
tima. 0 ancora, a realtà associative di tipo politico 0 all'ambiente professionale, ove l'ambizione e la volontà emulativa possono sfociare in rivalità e rancori di tipo aggressivo. La realizzazione di un delitto può poi generare da rapporti individuali di tipo amicale o sentimentale: il rapporto di intimità può infatti favorire il crearsi di circostanze vittimogene e la predisposizione all'aggressioni per moventi di tipo possessivo (ad esempio stalking e omicidi per gelosia 0 abbandono). I rapporti tra vittima e autore, infine, incidono anche sul piano giuridico costituendo talvolta il dato di genesi del reato (si pensi al delitto di violazione degli obblighi di assistenza familiare, ove il rapporto di parentela tra reo e vittima è elemento costitutivo del fatto), in altri casi, elementi di natura circostanziale 0 limiti di punibilità o procedibilità.

Vittima e processo penale italiano (cenni). Quanto alla posizione e alle tutele offerte alla vittima in sede processuale, viene in primo luogo in rilievo la Decisione Quadro del 15 marzo 2001 del Consiglio dell'Unione Europea, che ha previsto un articolato sistema di garanzie da adottare durante lo svolgimento del processo penale e nella fase successiva di applicazione della pena, individuando uno standard minimo di diritti che gli Stati membri devono necessariamente assicurare alle vittime di reati. In particolare, ex art. 2, ogni Stato deve garantire il riconoscimento della vittima nell'ambito del sistema giudiziario penale e il rispetto della sua dignità personale; questa deve essere sentita durante tutte le fasi del procedimento e interrogata solo se strettamente necessario (artt. 3 e 15); ha diritto ad essere adeguatamente informata relativamente alla tutela dei propri interessi, che vanno dalle modalità di presentazione della querela, all'assistenza legale, pur a spese dello Stato, e, più in generale, sullo svolgimento del processo (artt. 4 e 6); e deve esser rimborsata delle spese sostenute e risarcita dei danni patiti (artt. 7 e 9). Inoltre, è fatto obbligo agli Stati di proteggere la vittima e i suoi familiari da ogni eventuale forma di minaccia o ritorsione (art. 8). Per quanto concerne l'ordinamento italiano, la vittima, nonostante i passi in avanti operati dal nuovo codice, rimane per la verità un mero interlocutore occasionale nella dialettica processuale ordinaria, e vi partecipa solo in quanto persona offesa dal reato. Come soggetto processuale, gode comunque di facoltà di impulso e controllo dell'operato del pubblico ministero e del giudice: ha diritto, tra l'altro, a presentare querele 0 istanze, ad essere informata del compimento di atti irripetibili o della facoltà di nominare un difensore, e dispone di una serie di poteri sollecitatori e partecipativi. Inoltre, se ha patito un pregiudizio suscettibile di riparazione economica, può costituirsi parte civile e tutelare così la propria pretesa alle restituzioni 0 al risarcimento del danno ex artt. 74 ss. c.p.p.. Ad ogni modo, la valorizzazione della figura della vittima del reato trova i riscontri più significativi nei casi in cui sono ammesse forme di mediazione penale (si pensi al processo minorile) e più in generale con il ricorso alla cd. giustizia riparativa. Si consideri ad esempio il ruolo conciliativo attribuito al giudice di pace, funzionale a pervenire ad una soluzione del conflitto che possa anzitutto soddisfare la persona offesa. 0 alla facoltà offerta alla vittima dal D.Lgs. 274/2000, di ricorso immediato nei reati procedibili a querela: qui è evidente il riconoscimento di un autonomo spazio operativo nell'ambito del procedimento penale all'offeso, che può esercitare, con il coinvolgimento del pubblico ministero e del giudice di pace, una vera e propria forma di vocatio in iudicium rimessa alla sua disponibilità.

\section{Bibliografia}

A. Baldelli, Le vittime del reato nel processo penale, Torino, 2003.

A. Balloni, Vittime, crimine, difesa sociale, Bologna, 1989.

S. Barlati, M.E. Menini, M. Spagnolo, Dalla vittima all'autore del reato, Napoli, 2010.

R. Bisi, Vittime, vittimologia e società, in R. Bisi, P. Faccioli (a cura di), Con gli occhi della vittima. Approccio interdisciplinare alla vittimologia, Milano, 1996.

R. Bisi, Vittimologia. Dinamiche relazionali tra vittimizzazione e mediazione, Milano, 2004.

L. Bresciani, Persona offesa dal reato, in Dig. Pen., Torino, 1995, p. 527 ss.

M. Correra, D. Riponti, La vittima nel sistema italiano della giustizia penale, Padova, 1990.

G. Gulotta, La vittima, Milano, 1976.

A. Karmen, Crime victims: An introduction tovictimology, Cengage Learning, 2009.

G. Lopez, Victimologie, Parigi, 1997.

M. Monzani, Lezioni di psicologia criminale e vittimologia, Napoli, 1999.

E. Strina, S. Bernasconi, Persona offesa, parte civile, Milano, 2001.

G. Tranchina, La vittima del reato nel sistemapenale italiano, in G. Gulotta, Dalla parte della vittima, Milano, 1976.

E. Viano, Vittimologia oggi: i principali temi di ricerca e di politica pubblica, in A. Balloni, E. Viano (a cura di), IV Congresso Mondiale di Vittimologia. Atti della giornata bolognese, Bologna, 1989.

M. Vagaggini, Dalla parte della vittima, Milano, 1981.

\section{Dipendenza affettiva. Quando un sentimento diventa una droga Love addiction. When a feeling becomes a drug}

\section{Danila Pescina}

Psicologa, Psicoterapeuta, Criminologa; Specialista in Psicoterapia Breve ad Approccio Strategico. Esperta in Psicologia delle Dipendenze; Terapeuta EMDR; Docente Master Criminologia Università degli Studi Internazionali di RomaUNINT, Italy

Come scrive Erich Fromm ne "L'arte di amare" (1995) "il bisogno di amare è il bisogno fondamentale dell'uomo, superiore per urgenza a quello della fame, della sete o dello stesso "sesso", in quanto per soddisfarlo questi ultimi possono anche essere messi a tacere". Pertanto non vi sarebbe nessuna relazione, soprattutto d'amore, se non vi fosse anche un certo grado di dipendenza. Certo, l'amore deve rendere liberi, ma è proprio nel dosaggio tra libertà propria, condivisione e dipendenza sana dall'amore, dal benessere e dall'intimità con l'altra persona che si definisce un rapporto affettivo sereno, costruttivo e appagante. Vedremo quanto oggi sia molto difficile raggiungere questo equilibrio. Abbiamo bisogno dell'amore, ma ne siamo spaventati. $E$ così il nostro bisogno di condivisione, di appartenenza, di affetto e di amore può diventare una dipendenza da un affetto ed un amore dati (o ricevuti) in maniera sbagliata dalla persona sbagliata, con il rischio che il filo che ci lega all'amore diventi una catena da cui non riusciamo a liberarci. Lo studio delle dipendenze è un ambito di ricerca molto interessante sia dal punto di vista clinico sia per le molteplici interpretazioni eziologiche e sviluppi psicopatologici che ne possono scaturire. Nell'epoca in cui stiamo vivendo vanno sempre più diffondendosi quelle che vengono definite "dipendenze comportamentali", ovvero quelle dipendenze che non implicano l'uso di una sostanza e dove l'oggetto della dipendenza è un comportamento generalmente accettato dalla società. Con il termine inglese "addiction" si intende proprio la condizione di schiavitù rispetto a una sostanza, un comportamento, una relazione. Dunque possiamo considerare la dipendenza affettiva come una "droga senza l'uso di una sostanza”, o meglio, la sostanza diventa proprio l'oggetto d'amore da cui dipende in maniera esclusiva la propria felicità e infelicità. Nel corso della presentazione verranno esposti i risultati aggiornati di un'indagine qualitativa svolta su un campione di adulti di entrambi i sessi, attraverso l'utilizzo di un "Test diagnostico sulla dipendenza d'amore". A conclusione del presente lavoro vedremo quanto possa essere utile, sia come prevenzione che come cura, un percorso psicoterapeutico che aiuti il dipendente affettivo ad uscire dalla spirale della dipendenza.

\section{Bibliografia}

Alonso Fernandez F. Le altre droghe, Roma, Edizioni universitarie romane, 1999.

Carnes P.J. Don't call it love. Recovery from sexual addiction, Bantam Books, New York, 1992.

Fromm E. L'arte di amare, Mondadori, Milano, 1995.

Guerreschi C. New addictions. Le nuove dipendenze, Edizioni San Paolo, Milano, 2005.

\section{Uomini che odiano le donne. Donne che odiano gli uomini. Quando la fine di un amore può rovinare la vita Men who hate women. Women who hate men. When the end of love can ruin life}

\section{Danila Pescina}

Psicologa, Psicoterapeuta, Criminologa; Specialista in Psicoterapia Breve ad Approccio Strategico. Esperta in Psicologia delle Dipendenze; Terapeuta EMDR; Docente Master Criminologia Università degli Studi Internazionali di RomaUNINT, Italy

All'inizio si ama, alla fine si odia. Che cosa accade quando in una storia d'amore non c'è il lieto fine? Dove finisce l'amore che si era provato per il coniuge? Sempre più di frequente la fine di una relazione può rovinare la vita. Secondo i dati Istat relativi all'anno 2015, anno in cui è stato introdotto anche il cosiddetto "divorzio breve", vi è stato un consistente aumento del numero di divorzi, che ammontano a $82.469(+57 \%$ sul 2014) e di separazioni pari a 91.706 (+2,7\% rispetto al 2014). Non tutti i divorzi o separazioni, fortunatamente, sono conflittuali. Ma se la coppia ha dei figli la probabilità che le ostilità, soprattutto per l'affido e/o il collocamento del figlio, aumentano in maniera esponenziale. Troppo spesso, accecati da rabbia e rancori coltivati per anni all'interno della relazione, queste coppie arrivano alla separazione con odio accecante nei confronti dell'ex coniuge pronti a far la guerra con tutti i mezzi possibili a disposizione. L'arma migliore per ferirsi diventano i figli, i quali diventano le vere vittime dell'odio dei genitori, tanto che, sempre più spesso, sono chiamati ad intervenire i Servizi Sociali e i CTU per 
cercare di trovare un accordo diversamente attuabile. Figli che si trovano improvvisamente a doversi schierare a favore di uno o dell'altro genitore, a dover compiacere 0 a dover addirittura mentire pur di non deludere il genitore collocatario. Tutto questo a discapito della sana crescita affettiva ed emotiva del bambino. Non possiamo non ricordare che assistere alla violenza di un genitore verso l'altro crea confusione nel mondo interiore dei bambini su ciò che è affetto, intimità, violenza e va a minare il cuore delle relazioni primarie. Quindi non è solo la violenza fisica assistita che crea dei traumi nel bambino, ma anche la violenza psicologica a cui deve assistere e, più o meno consapevolmente, farne parte, che crea enormi problemi nello sviluppo psichico del minore. Vedremo quindi, attraverso dei casi, quanto le separazioni conflittuali possano causare gravi danni alle madri, ai padri e soprattutto ai figli.

\section{Prevenire e ridurre le dipendenze nella pre-adolescenza: il Coping Power Program \\ Preventing and reducing addictions in the pre-adolescence: The Coping Power Program}

Gennarina Pirri

Psicologa Clinica, Criminologa, Psicoterapeuta Cognitivo Comportamentale. Terapeuta EMDR, Trainer Coping Power Program clinico, Trainer Coping Power Scuola.; Referente Psicologo regione Friuli Venezia Giulia AIFA (Associazione Italiana Famiglie ADHD), Italy

Le dipendenze da stupefacenti e il comportamento delinquenziale rappresentano gravi problemi che affliggono la salute degli adolescenti e compromettono il loro adattamento sociale (Viner e Barker, 2006). KimCohen et al., nel 2003 hanno affermato l'importanza dei trattamenti durante l'infanzia, poiché questi possono servire da strumento di prevenzione e portano ad una riduzione nell'uso di sostanze, che aumenta rapidamente durante l'adolescenza. Un programma di prevenzione e trattamento che risponde a queste esigenze è il Coping Power Program (Lochman e Wells, 1996), tradotto e validato anche in Italia (Muratori P., et al., 2012). II CPP è un intervento multimodale che si basa sul Contextual Social-Cognitive Model (Lochman e Wells, 2002). La componente per i bambini (32 sessioni), prevede l'utilizzo di tecniche cognitivocomportamentali e attività volte al potenziamento di diverse abilità, quali intraprendere obiettivi a breve e lungo termine, organizzare efficacemente lo studio, riconoscere e modulare i segnali fisiologici della rabbia, riconoscere il punto di vista altrui (perspective taking), risolvere adeguatamente le situazioni conflittuali, resistere alle pressioni dei pari ed entrare in contatto con gruppi di pari positivi. Inoltre è previsto l'utilizzo di contratti comportamentali (i traguardi) in cui vengono stabiliti obiettivi minimi scolastici o sociali, al cui raggiungimento è associato un sistema a premi (tecnica della Token Economy). II role-playing e l'interazione con i pari sono i principali strumenti utilizzati dal programma allo scopo di incrementare la generalizzazione delle competenze acquisite al di fuori del setting terapeutico. La componente per i genitori (16 sessioni) ha l'obiettivo di sviluppare e potenziare le abilità genitoriali relative a diverse aree quali la promozione dell'organizzazione delle abilità di studio, la modulazione dello stress genitoriale, l'utilizzo di appropriate pratiche educative, l'incremento della comunicazione familiare e la progettazione di momenti di condivisione con i figli. Le sessioni si portano avanti in modo parallelo. Gli effetti preventivi del CPP sulla delinquenza e la dipendenza da stupefacenti sono stati individuati in diversi studi. In uno studio su 183 adolescenti aggressivi (9-11 anni), il CPP ha prodotto riduzioni nel comportamento delinquenziale e nell'uso di sostanze secondo quanto confermato dai rispettivi genitori ad un anno dal trattamento, in comparazione con un gruppo di controllo non sottoposto ad alcun trattamento (Lochman e Wells, 2002-2004). In un altro studio che ha coinvolto 243 giovani di ambo i sessi di età compresa tra i 10 e 11 anni, il CPP ha prodotto una riduzione nell'uso di sostanze ed un miglioramento nella competenza sociale, nell'autoregolazione, e nel coinvolgimento genitoriale (Lochman e Wells, 2002). Anche questo studio, come il precedente ha confermato questi risultati dopo un anno dal trattamento (Lochman e Wells, 2003). Un ulteriore studio (Marjo JS., et al., 2007) è stato effettuato ad Utrecht su bambini con diagnosi di DCD, (8-13 anni). Sono stati assegnati casualmente ad una terapia comportamentale manualizzata, adattata partendo dal CPP (L'Utrecht Coping Power Program; UCPP) e ad un gruppo di controllo (CU) sottoposto ad altri tipi di trattamenti preesistenti. Cinque anni (2003-2005) dopo l'inizio del trattamento (1996-1999), sono stati monitorati uso di sostanze e delinquenza in 61 dei 77 adolescenti iniziali ed i risultati sono stati comparati con un gruppo di controllo di soggetti sani (HC), attraverso questionari self-report. (II CSAP Student Survey, Pentz et al., 1989, e il National Youth Survey, Elliott et al., 1985).
Dai risultati emerge che a lungo termine, $i$ tre gruppi non differiscono per quanto riguarda l'uso di alcool e sigarette ma differiscono nell'uso di marijuana ( $x 2=7.5 ; p=.023)$, con molti adolescenti del gruppo CU che fanno uso di marijuana se comparati agli adolescenti del gruppo UCPP $(C U=35 \%, U C P P=13 \% ; x 2=4.0, p<0.045)$ e molti più adolescenti $C U$ che fanno uso di marijuana in comparazione con adolescenti del gruppo $\mathrm{HC}$ $(\mathrm{CU}=35 \%, \mathrm{HC}=13 \% ; \mathrm{x} 2=6.3, \mathrm{p}<.01$ ). Dai risultati di questi studi sembra che il CPP e L'UCPP siano più incisivi rispetto ad altri tipi di trattamento nel ridurre le dipendenze da stupefacenti nella pre-adolescenza. Nello specifico per quanto riguarda l'uso di sostanze l'UCPP ha portato i suoi partecipanti nel range del campione dei soggetti sani (13\%). Di conseguenza, una più capillare diffusione del CPP nelle strutture sanitarie italiane porterebbe a più efficaci forme di prevenzione e trattamento dei disturbi esternalizzanti e delle diverse forme di dipendenze ad esso associate, oltre ad offrire un risparmio significativo in termini economici e di costi per il Servizio Sanitario Nazionale, grazie alla necessità di applicare il protocollo in forma di gruppo.

\section{Bibliografia}

Elliott D.S., Huizinga D., Ageton S.S. (1985), Explaining Delinquency and Drug Use. Beverly Hills, CA: Sage Publications.

Kim-Cohen J., Caspi A., Moffitt T.E., Harrington H., Milne B.J., Poulton R. (2003), Prior juvenile diagnoses in adults with mental disorders: developmental follow-back of a prospective-Iongitudinal cohort. Arch Gen Psychiatry 60:709Y717.

Lochman J.E., Wells K.C. (1996), A social-cognitive intervention with aggressive children: prevention effects and contextual implementation issues. In: Preventing Childhood Disorders, Substance Abuse and Delinquency, Peters R, McMahon R, eds. Thousand Oaks, CA: Sage Publications, pp 111 Y143.

Lochman J.E., Wells K.C. (2002), Contextual social-cognitive mediators and childoutcome: a test of the theoretical model in the coping power program. Dev Psychopathol, 14-4, pp. 945-67.

Lochman J.E., Wells K.C. (2002b), The Coping Power Program at the middle- chool transition: universal and indicated prevention effects. Psychol Addict Behav 14:S40YS54.

Lochman J.E., Wells K.C. (2003), Effectiveness study of Coping Power and classroom intervention with aggressive children: outcomes at a one-year follow-up. Behav Ther 34:493Y515.

Lochman J.E., Wells K.C. (2004), The Coping Power Program for preadolescent aggressive boys and their parents: outcome effects at the 1-year follow-up. J Consult Clin Psychol 72:571Y578.

Marjo J.S., Zonnevylle-Bender, Walter Matthys, MD, Nicolle M.H., Van de Wiel, John E. Lochman (2007), Preventive effects of treatment of distruptive behavior disorder in middle childhood on substance use and delinquent behavior. J. AM. Acad. Child Adolesc. Psychiatry, 46:1.

Muratori P. et al. (2012), Coping Power. Programma per il controllo di rabbia ed aggressività in bambini e adolescenti, Erikson, Trento (ed. or. J. Lochman, K. Wells, L. Lehart, Coping Power Program, Guilford Press, Oxford). Pentz MA, Dwyer JH, Mackinnon DP et al. (1989), A multicommunity trial for primary prevention of adolescent drug abuse: effects on drug use prevalence. JAMA 261:3259Y3266.

Viner R.M., Barker M. (2006), Young people s health: the need for action. BM 330:901Y903

\section{Le coppie criminali. Dalla "folie à deux" al vizio di mente: una disamina di delitti efferati perpetrati in coppia Criminal couples. From "madness shared by two" to the mental illness: A review of brutal crimes performed in pairs}

Francesca Righi

Dottoressa in Giurisprudenza, Criminologa, Grafologa Forense in formazione, Università “Sapienza” Roma, Italy

La coppia criminale è un fenomeno poco comune, poiché i criminali agiscono da soli o coinvolgendo altri individui in maniera fortuita. Gli omicidi commessi in coppia rappresentano un'eventualità piuttosto rara (si stima che in Italia siano all'incirca il 5\%). Alla base di questa particolarissima dinamica vi è la c.d. folie à deux, altrimenti indicata nella nosografia psichiatrica come "Disturbo Psicotico Condiviso e dall'ICD-10 come "Disturbo Delirante Indotto". Descritto per la prima volta nel 1887 da Lasègue e Falret, esso si caratterizza per la comparsa di un delirio in un soggetto detto Caso Primario, condiviso dal soggetto indotto. La coppia delirante vive in stretta correlazione e al contempo isolata dal contesto sociale, condizioni che facilitano l'influenza dell'incube, portatore di una patologia mentale più grave, sul succube non necessariamente affetto da una patologia psicotica, né, per forza proveniente da una subcultura criminale. II succube dunque accoglie le idee deliranti dell'incube e le fa proprie, dando vita non alla semplice 
somma di due individui ma ad un quid novi rappresentato dalla coppia, unita da una dipendenza patologica fortissima. Tale contributo, ha il fine di evidenziare attraverso l'analisi di alcune famose coppie omicide, il riconoscimento o meno, di un vizio totale o parziale di mente e la eventuale imputabilità, ivi incluse le considerazioni sul reinserimento sociale degli stessi, una volta espiata la pena detentiva.

\section{Bibliografia}

Fornari U. Trattato di psichiatria forense, Utet, Torino, 1997.

Furio J. Team Killers. A comparative study of collaborative criminals, Algora Publishing, New York, 2001.

ICD-10, Classification of Mental and Behavioral Disorders: clinical descriptions and diagnostic guidelines, Ed. italiana a cura di D. Kemali, M.May, F.Catapano, S.Lobrace, L. Magliano, Masson, Milano, 1992.

Lasegue C., Falret J. La folie à deux (ou folie communiqueè), Annales MedicoPsycologique, vol. 18,1877.

Marini G. Incapaci (circonvenzione di), in Dig. pen., Vl, Torino, 1992, 308 ss.

Mastronardi V. Manuale per operatori e psicopatologi forensi, Giuffrè, Milano 2012.

Mastronardi V., De Luca R. I serial killer. Chi sono e cosa pensano? Come e perché uccidono? La riabilitazione è possibile? Newton \&Compton Editori, Roma, 2005.

Mastronardi V., L.M., Ventura N. Imputabilità, coscienza morale e psicopatologia. Profili comparatisti internazionali, Riv Psichiatr, 47(4 Suppl.1), 2012, pp.17s-25s.

Palermo G. B., Mastronardi V. Il profilo criminologico, Dalla scena del crimine ai profili socio-psicologici, Giuffrè, Milano, 2005.

\section{Scena del crimine: staging e procurato allarme Crime scene: Staging and terrifying people/false police report}

Michelangelo Russo

Avvocato Penalista del Foro di Cosenza, Iscritto all'Albo Speciale per il Patrocinio davanti alle Giurisdizioni Superiori, Italy

Non sono mai mancati e, c'è da crederlo, non mancheranno mai, soggetti che delinquono non solo ponendo in essere, com'è ovvio, comportamenti vietati dal Codice Penale (un libro questo che, mi è sempre piaciuto dire, più che regolare le azioni umane, regola la vita della intera Società), ma che delinquono anche "inventandosi" di sana pianta l'esistenza di fattispecie penalmente sanzionate. È proprio per questo motivo, infatti, che il Legislatore penale del 1930 ha inserito all'interno del terzo libro del codice penale, all'articolo 658, il reato contravvenzionale del, cit.: "Procurato allarme presso l'Autorità". Più precisamente, l'art. 658 c.p., recita: "Chiunque, annunziando disastri, infortuni o pericoli inesistenti, suscita allarme presso l'Autorità, o presso enti o persone che esercitano un pubblico servizio, è punito con l'arresto fino a sei mesi 0 con l'ammenda da euro 10 a 516". Dalla lettura del testo dell'articolo si ricava subito che siamo in presenza di un reato oblabile ex art. 162-bis c.p. (c.d. oblazione discrezionale), in quanto il fatto vietato viene punito alternativamente con l'arresto o con l'ammenda. Pertanto, per la fattispecie penale incriminatrice non è prevista l'oblazione semplice di cui all'art. 162 c.p. II bene giuridico tutelato dalla norma penale è l'ordine pubblico, il quale deve essere garantito nei confronti delle condotte di falso allarme sociale che sono portate a conoscenza dell'Autorità 0 di altri enti esercenti un servizio di pubblica necessità. La fattispecie penale in oggetto ha natura plurioffensiva, poiché è idonea a ledere la pubblica e la privata tranquillità, così come anche il buon andamento, l'efficienza e l'efficacia dell'attività della pubblica amministrazione e, altresì, costituisce reato di pericolo. Nella pratica quotidiana ciò significa, dunque, che basta la mera diffusione di una falsa notizia o di un falso allarme, idonei da soli a far scattare le procedure delle autorità per un pericolo che, in realtà, è inesistente, a configurare l'ipotesi di reato del procurato allarme. Un reato, perciò, che si configura con la sola idoneità dell'annuncio a creare allarme. È questo il caso che si verifica, per esempio, allorquando si richiede, mediante una denuncia, l'intervento delle Forze dell'Ordine, per un reato che determina un dispiegamento di uomini e mezzi per contrastare le conseguenze di un fatto punito dall'Ordinamento, tuttavia in realtà mai verificatosi. II reato in parola può essere commesso da chiunque, ma costituisce un fatto innegabile che alcune categorie di soggetti sono facilitate più di altre nel commetterlo, come ad es. un giornalista che pubblica (oppure confida all'Autorità) la notizia di un possibile attentato contro un esponente istituzionale. Non incorre in questo reato, invece, il giornalista che pubblica una notizia falsa (ma da egli ritenuta verosimilmente plausibile), che sia stata raccolta da una fonte identificata e attendibile, sempre che sia stata pubblicata adoperando il condizionale. Vale a dire, cioè, che la notizia sia stata data mettendone però in dubbio la sua veridicità. In altri termini, deve potersi desumere, per scriminare il reato, il fatto che da indagini di polizia fosse possibile capire che quella notizia era falsa; in tal caso il giornalista non incorrerebbe nel reato di procurato allarme. Secondo l'orientamento prevalente e consolidato nel tempo della Suprema Corte di Cassazione, infatti, il giornalista, prima di pubblicare una notizia, ha l'obbligo professionale di accertare la veridicità della stessa, tanto più se la notizia è di particolare gravità e idonea a suscitare allarme non solo nella pubblica opinione, ma anche nelle autorità preposte alla tutela dell'ordine pubblico. È peraltro evidente che, qualora una notizia non sia verificata e/o almeno verificabile, non deve essere pubblicata. Tanto più se la notizia è idonea da sola, per come detto, a suscitare pubblico allarme presso le Autorità. Viceversa, non integra il reato di cui all'art. 658 c.p. la condotta di chi segnala falsamente, col mezzo del telefono alla polizia giudiziaria, sollecitandone l'intervento, il fatto che il coniuge gli impedisca di incontrare il figlio minore, in quanto con detto comportamento non si prospetta un pericolo presso l'Autorità tale da ingenerare pubblico allarme. Questo perché il fatto non è tale da dare luogo ad una situazione, appunto, di allarme pubblico ma, al massino, è idoneo soltanto a turbare la quiete domestica. Rientra invece a pieno titolo nell'ipotesi di reato in questione il fatto di colui il quale crei una situazione di panico generalizzato, che richiede l'intervento delle forze dell'ordine. Ne sono esempi: fare scoppiare un petardo in mezzo alla folla, gridando che è in corso un attentato, o quanto accaduto nel giugno del 2017 a Navacchio, in provincia di Pisa, dove tre giovani (di 26, 27 e 35 anni) hanno inscenato il rapimento di un loro amico (che era d'accordo), prossimo alle nozze, nel parcheggio di una zona commerciale, di sabato mattina, con tanto di colpi di pistola (in realtà, due colpi di scacciacani, sparati in aria) e morto (ovviamente finto) sull'asfalto e, dulcis in fundo (recte: amarus in fundo), vero intervento dei Carabinieri del Comando Provinciale di Pisa, ai quali non è stato certo semplice spiegare che si trattasse di uno staging della scena del crimine ioci causa (infatti, i tre burloni, in stile "Amici miei", sono stati poi denunciati per procurato allarme). La diffusione di notizie false - che, tuttavia, un tempo era, e del resto poteva essere solo ed esclusivo appannaggio dei giornalisti -, oggi viceversa costituisce, come spesso nella esperienza quotidiana di ognuno di noi è facile ravvisare, un problema di non poco conto; è appunto il caso di dire di vero e proprio "allarme sociale", stante la diffusione dei social network e degli altri nuovi mezzi di comunicazione, come la nota applicazione per telefonia mobile denominata "Whatsapp". L'uso, infatti, sempre più disinvolto, ormai, quando non addirittura criminale, dei nuovi mezzi di comunicazione "social", consente a chiunque in pratica di innescare la miccia del pericolo (inesistente) con una facilità e velocità d'esecuzione mai conosciute prima. Ognuno, infatti, può facilmente consumare (a volte anche inconsapevolmente, come nel caso delle c.d. "fake news") su Facebook, il reato in discorso, diffondendo notizie e/o informazioni false 0 che, perlomeno, si rivelano poi alla prova dei fatti puntualmente infondate. In conclusione, sarebbe auspicabile a fini di un valido contrasto dei fatti, posti a base della contravvenzione di cui all'art. 658 c.p., una vera e propria legislazione specifica che, inasprendo, da una parte, le (blande) pene attualmente previste per il reato in parola, introduca, dall'altra, anche il divieto temporaneo dell'uso dei social, e degli altri moderni mezzi di comunicazione, da parte di chi in maniera irresponsabile lede il bene tutelato dalla norma.

\section{Bibliografia}

Canzio G. (2010), Art. 658 c.p. Procurato allarme presso l'Autorità, in Codice penale, Rassegna di giurisprudenza e di dottrina, XIII, a cura di Giorgio Lattanzi ed Ernesto Lupo, Giuffrè, Milano.

L'Insalata M. (2012), Procurato allarme presso l'Autorità (art. 658), in Trattato di diritto penale. Parte speciale, a cura di Roberto Cadoppi, UTET, Torino.

Manzini V. (1984), Art. 658 c.p. Procurato allarme presso l'Autorità, in Trattato di diritto penale italiano, $X$, UTET, Torino.

Pisa P. (2009), Reati contro l'amministrazione della giustizia, in Trattato di diritto penale, diretto da C.F. Grosso, T. Padovani, A. Pagliaro, Giuffrè, Milano.

\section{La vittimizzazione delle prostitute nigeriane tramite la ritualità JuJu: meccanismi psicologici e basi antropologiche \\ The Juju rite as a means to victimize the prostitutes from Nigeria: Psychological mechanisms and anthropological bases}

\section{Giuseppe Gino Saladini}

Specialista in Medicina Legale, Specialista in Psicologia Clinica, Criminologo, Docente Unitelma Sapienza Roma, Docente Master in Criminologia, Sistema Penale, Psicopatologia Forense, Investigazioni e Sicurezza UNINT Università degli Studi Internazionali di Roma, Italy

Il traffico di donne nigeriane destinate al mercato del sesso mercenario 
italiano ha avuto inizio verso la fine degli anni ottanta e si è mantenuto costante fino ad oggi, tanto che attualmente l'Italia è la principale nazione europea di destinazione. L'organizzazione della rete criminale della mafia nigeriana si caratterizza per delle peculiarità sia nei modelli coercitivi, sia per le modalità di reclutamento e per le figure che ruotano attorno alla tratta e al successivo sfruttamento sessuale delle giovani donne. La mafia nigeriana si articola attorno ad un network costituito quasi interamente da elementi appartenenti allo stato di origine e, a differenza delle organizzazioni criminali dei paesi dell'Europa dell'est, che usano la violenza come strumento coercitivo, si basa su un vero e proprio patto, sancito attraverso elementi religiosi legati alla cultura e alla religiosità tradizionale. Ragazze tra i 14 e i 22 anni di età appartenenti a famiglie estremamente povere, vengono reclutate e, con la falsa promessa di un lavoro da baby sitter o da parrucchiera in Italia, sono invitate a contrarre un debito per avere i fondi per raggiungere il nostro paese, con l'impegno a restituire il denaro, una volta giunte a destinazione, grazie al lavoro che svolgeranno. II contratto è suggellato da riti magici svolti in Nigeria prima della partenza. Si tratta di riti JuJu (un misto di rito voodoo impregnato da giuramenti e sottomissioni), che hanno un impatto fortissimo sulle ragazze, che ne subiscono la forza coercitiva, scoraggiando una loro eventuale fuga. Le ragazze sono spesso analfabete o con scarsa istruzione e non capiscono che la cifra che dovranno restituire è in euro e non in naira, la moneta locale, quindi pensano sia abbordabile. Quando accettano vengono condotte davanti ad uno stregone juju che celebra il rito. Si tratta di un maleficio praticato attraverso tagli sulla pelle delle giovani, che poi che vengono ricoperti di cenere. II rituale viene completato prelevando alle ragazze una ciocca di capelli, frammenti di unghie delle mani e la biancheria intima (il materiale sarà poi conservato dallo stregone come feticcio su cui attivare, inseguito, un eventuale maleficio a morte). II rito termina con l'uccisione di un gallo di cui le ragazze sono costrette a ingerire il cuore insieme ad una bevanda alcolica. Una volta arrivate in Italia le giovani donne trovano ad attenderle una madam (o maman) che continua a suggestionarle e a condizionarle. Molto spesso si tratta di un'ex prostituta, a suo tempo vittima di tratta e riscattatasi tramite il pagamento del debito. La madam è colei che riscuote i soldi per saldare il debito e che fa da tramite con la mafia nigeriana. Le ragazze incarnano una tipologia di vittima incapace di liberarsi del giogo criminale e, quindi, di denunciare i loro aguzzini, perché sono terrorizzate dal rito cui sono state sottoposte. C'è molto sincretismo, tutte credono nel Dio cristiano 0 in Allah, divinità che sono più forti di ogni malocchio, ma la religione tradizionale prende il sopravvento, alimentata dall'azione vittimizzante della madam che le sottopone a un vero e proprio brainwashing, con un indebolimento dell'autonomia personale, determinando una situazione d'incapacità di pensare autonomamente e attuando un sistematico rinforzo delle credenze legate al rito JuJu cui sono state sottoposte in Nigeria. La madam è vissuta dalle vittime come un'aguzzina e, al tempo stesso, come una figura degna di rispetto. Le ragazze che riescono a pagare il loro debito sono costrette a organizzare una festa rituale di liberazione. II lavoro presentato, che si basa su ricerche di etnopsichiatria e su interviste con giovani ex prostitute e con alcune ex maman, ascoltate nell'ambito di una ricerca preparatoria a una docufiction sulla mafia nigeriana, illustra la modalità di vittimizzazione tramite la ritualità religiosa illustrandone i meccanismi psicologici e antropologici.

\section{Bibliografia}

Adarabio I. II coraggio di Grace: donne nigeriane dalla prostituzione alla libertà, Prospettiva Edizioni, Roma 2003.

Aikpitanyi I. 500 Storie vere sulla tratta delle ragazze africane in Italia, Ediesse, Roma, 2011.

Bernardotti A., Carchedi F., Fiore B. Schiavitù emergenti. La tratta e lo sfruttamento delle donne nigeriane sul litorale domitio. Ediesse, Roma, 2005.

Carchedi F. La tratta delle minorenni nigeriane in Italia. I dati, i racconti, i servizi sociali Associazione Parsec, UNICRI, Roma, 2010.

Coppo P. Guaritori di follia, Boringhieri editore, Torino, 1994.

Coppo P. Etnopsichiatria, II saggiatore editore, Milano, 1999.

Davis M.D.J. Parlo di me (senza paura), Amazon Independently published, 2017.

DSM-5, Manuale Diagnostico e Statistico dei Disturbi Psichiatrici (Edizione italiana a cura di M. Biondi), Milano, Ed. Raffaello Cortina, (prima ed.), 2014.

Lévi -Strauss C. II pensiero selvaggio, II saggiatore editore, Milano, 1964.

Lévi -Strauss C. Lo stregone e la sua magia, II saggiatore editore, Milano, 1964.

Mastronardi V.M. Criminologia Clinica: in tema di vittimologia, prevenzione e trattamento, Edizioni Universitarie, Roma, 2008.

Mirabile R. La mano nera sulla città, IIMostardino\&CaramellaBuona editore, Roma, 2016.

Nathan T. Principi di etnopsicanalisi, Torino, Bollati Boringhieri, 1996.

\section{Disney: il crimine nelle favole Disney: Crime in fairy tales}

Marta Senesi

Dottore in Scienze e Tecniche Psicologiche. Criminologa. Grafologa in formazione presso la Sapienza Università di Roma; Componente Istituto Internazionale di Scienze Criminologiche e Psicopatologico Forensi (IISCPF); Tutor presso il Master di Criminologia Università degli Studi Internazionali di Roma UNINT, Italy

Lo schermo si colora di un celeste cielo, e comincia a delinearsi un castello sotto la cui bianca aurea si staglia la sigla Walt Disney Pictures, un semiarco corona il tutto e le porte della fantasia sono pronte a schiudersi per gli spettatori. Questa è la classica presentazione di un Film Disney a cui almeno una volta nella vita sarà capitato a ciascuno di noi di assistere, e che accompagna l'infanzia di milioni di bambini. Ma cosa ci aspetta dietro quel castello? Affascinanti incantesimi e scintille di magia? Senz'altro. Principesse da salvare e principi azzurri da sognare? Assolutamente sì. Animali parlanti e un'isola che non c'è? Tutto questo e molto di più. Se guardassimo, però, quel castello da una prospettiva diversa, e se spostassimo l'attenzione dal vestito prezioso della protagonista, a ciò che motiva in profondità le sue scelte e alle dinamiche che la circondano, potremmo notare delle sfumature oscure, celate da sfavillanti gioielli ed innaturali tramonti, tanto brillanti da accecarci. È proprio su quelle sfumature più tetre che si concentrerà il lavoro, realizzato insieme alle Dottoresse Monica Calderaro e Danila Pescina, in cui tramite l'analisi e l'approfondimento di scene e temi dei Film Disney sarà possibile riscontrare aspetti criminologici e psicopatologici, cercando così di connettere due mondi tanto distanti che per un attimo si intersecheranno, cosi come in una tavolozza di un pittore è possibile mescolare il bianco e il nero. Uno dei principali film considerati è la Bella e la Bestia, in cui la protagonista Belle, innamorandosi del suo sequestratore durante un periodo di prigionia, è chiaramente affetta dalla sindrome di Stoccolma. In Biancaneve, invece, ad essere macabra è la bizzarra condotta del Principe, che non appena viene a conoscenza della fanciulla che giace morta nel bosco, corre da lei e la bacia, un vero e proprio atto carnale su di un corpo privo di vita, che prende il nome di necrofilia. Inoltre, in una celebre scena de "La Spada nella Roccia", Mago Merlino e Semola, tramutati in scoiattoli, sono inseguiti e tormentati dalle attenzioni di scoiattoline che, innamoratesi di loro, sono convinte che il proprio sentimento sia corrisposto, portando in scena una chiara forma di Delirio Erotomane. A dispetto di qualsiasi aspettativa fiabesca e surreale, il presente lavoro ha portato in scena svariate realtà piuttosto drammatiche e molto poco romantiche, senza tralasciare dinamiche ricorrenti, come quelle ipnotiche che, nonostante siano semplicisticamente ridotte ad un tocco di magia, appartengono, in verità, ad entità assolutamente più complesse e tetre. L'intento, però, non è certo quello di adombrare simili capolavori, anzi, questa miscela esplosiva di bene e male è ciò che li rende assolutamente ancor più affascinanti e degni di essere guardati almeno una volta nella vita, non solo dai bambini, ma anche e soprattutto dagli adulti.

\section{Bibliografia}

Aggrawal A. (2009), A new classification of necrophilia, Journal of Forensic and Legal Medicine, 16, pp. 316-320.

Favaro A., Degortes D., Colombo G. (2000), The effects of trauma among kidnap victims in Sardinia, Psychological Medicine, 30(5), pp. 975-980.

Graham D.L., Rawlings E.I. (1995), A scale for identifying Stockolm Syndrome reactions in young dating women: factor structure, reliability and validity, Violence Victims, 10(3), pp. 3-22.

Segal J.H. (1989), Erotomania revisited, Am J Psychiatry, 146(10), p. 1261.

\section{La giustizia riparativa nella prospettiva vittimologica integrata. Art. 162-ter c.p. \\ Restorative justice in the integrated criminological perspective. Law Article 162-ter c.p.}

Martina Vallesi

Giurista, Criminologa, Italy

Nonostante gli sforzi profusi dai cultori delle scienze criminologiche e vittimologiche, il processo penale italiano sembra essere una partita a due: da un lato abbiamo l'autore del reato, dall'altro lo Stato, in mezzo c'è il bene giuridico leso dal reato e.. la vittima? Alla vittima non rimane che godersi lo spettacolo e, subirne gli effetti senza poter incidere sulle sue sorti. Ė necessario partire da fatti pacifici. Ed è pacifico che esiste l'autore del reato, così come esiste la vittima, e ciò, perché il reato è interazione; è una conflittualità sorta all'interno di un rapporto di tensione personale nella diade reo/vittima; il reato non è solo un 
torto alla società, ma anche violazione dei diritti individuali delle vittime. Altresì pacifica, sull'argomento, è la sensibilità mostrata dalla comunità internazionale ed europea. Appurato come il processo possa danneggiare ulteriormente la vittima, più di quanto non lo abbia fatto il reato, già nel 1985 il Consiglio d'Europa raccomandava agli Stati membri di tutelare le vittime in tutte le fasi del procedimento, onde prevenire episodi di vittimizzazione secondaria. Constatata, tuttavia, la perdurante inottemperanza alle varie Risoluzioni, Raccomandazioni, Decisioni quadro, via via succedutesi nel tempo, nel 2012, I'Unione Europea interviene con la Direttiva 2012/29/UE recante "norme minime in materia di diritti, assistenza e protezione delle vittime di reato", al fine di riportare i riflettori sulla vittima di reato. Con tale atto, non si introduce un soggetto processuale autonomo ma, si consente alla vittima di interloquire con le altre parti processuali, prima, durante e, dopo il procedimento penale, riconoscendo una serie di diritti, quali, il diritto di ricevere informazioni ad essa comprensibili, il diritto alla partecipazione processuale, il diritto alla speciale protezione dovuta alla condizione di particolare vulnerabilità, il diritto all'assistenza; tutte garanzie volte a scongiurare la vittimizzazione secondaria e ripetuta, le intimidazioni e le ritorsioni. Infine, ed è questo il tema più caro all'Europa, le garanzie di accesso alla restorative justice, una giustizia relazionale con il compito garantire la risoluzione stragiudiziale del conflitto tra vittima e autore di reato e, di riaprire i canali comunicativi interrotti dall'evento negativo, attraverso la mediazione, il dialogo esteso ai gruppi parentali ed i consigli commisurativi; uno strumento di cui avvalersi per spingere la pena all'auspicato ruolo di extrema ratio e riposizionare la vittima al centro dell'attenzione. E, I'Italia cosa fa? Intanto, il recepimento della Direttiva, mediante D.Lgs. 212/2015, si è avuto in ritardo e, dunque, lasciamo al beneficio del dubbio quanto questa predisposizione "benevola" dello Stato italiano nei confronti della vittima sia stata spontanea o quanto, invece, finalizzata ad evitare una procedura per infrazione per violazione degli obblighi comunitari; in secondo luogo, l'adeguamento è avvenuto mediante una sorta di convalida per trasposizione del contenuto della normativa comunitaria in quella nazionale, senza nessun investimento innovativo, omettendo, persino, qualsivoglia riferimento ai servizi di giustizia riparativa. In ultimo, addirittura, il legislatore ha fatto ricorso ad una serie di istituti a carattere deflativo, dei quali, il più recente è l'art. 162-ter c.p., che consente l'estinzione del reato per condotte riparatorie, che di riparativo non ha nulla, se non il nomen. Una norma che, ancora una volta, dimostra l'ipertrofia normativa in cui versa il nostro sistema penale, generatrice di una schizofrenia ormai insanabile. Un ordinamento che con una mano dà, e con l'altra toglie. Da un lato, garantisce alla persona offesa il diritto di difendersi mediante proposizione di querela, dall'altro, glielo sottrae per affidarlo al giudice, a beneficio del reo e del sistema penale. La verità è che il criminale fa paura; la vittima resta un soggetto inoffensivo cui riconoscere, nella migliore delle ipotesi, il risarcimento del danno.

\section{Bibliografia}

Amato G. Adesso l'imputato può "cancellare" la sua condotta illecita, in Guida al Diritto, n. 31,2017

Ambrosetti E.M. La riforma Orlando: profili di diritto penale sostanziale, in Processo Penale e Giustizia, 2017.

Baldelli A. Le vittime del reato nel processo penale, Torino, 2003.

Balloni A. Vittime, crimine, difesa sociale, Clueb, Bologna, 1989.

Bene T. La persona offesa tra diritto di difesa e diritto alla giurisdizione: le nuove tendenze legislative, in Archivio Penale, 2013.

Correra M. Riponti D. La vittima nel sistema italiano della giustizia penale, Padova, 1990.

Del Tufo M. La tutela della vittima in una prospettiva europea, in Diritto Penale e Processo, 1999.

Giostra G. Il processo penale minorile. Commento al D.P.R. 448/1988, Milano, 2007.

Guerra S. L'estinzione del reato conseguente a condotte riparatorie, in Scalfati A., II giudice di pace. Un nuovo modello di giustizia penale, Padova, 2001.

Gulotta G. La vittima, Giuffré, Milano, 1976.

Lopez G. Victimologie, Parigi, 1997.

Mancini S. Diritto penale internazionale, a cura di Mezzetti E., Giappichelli, Torino, 2010.

Mannozzi G. La giustizia senza spada. Uno studio comparato su giustizia riparativa e mediazione penale, Giuffré, Milano, 2003.

Mastronardi V., Ricci S., De Vita L., Pomilla A. Relazione tra offender e vittima dalle rivelazioni di uno stupratore seriale e delle sue vittime, in Rivista di Criminologia, Vittimologia e Sicurezza, Vol.VI, n. 3, 2012.

Monzani M. Lezioni di psicologia criminale e vittimologia, Napoli, 1999.

Neri G. Elementi di criminologia, Nuova Cultura, Roma, 2012.

Rossi G. La Direttiva 2012/29/UE: vittima e giustizia riparativa nell'ordinamento penitenziario, in Archivio Penale, n. 2, 2015

Rossi G. La giustizia riparativa nel procedimento penale minorile alla luce della direttiva 2012/29/UE, in Rassegna penitenziaria e Criminologica, n. 1, 2015

Scardaccione G. Nuovi modelli di giustizia: giustizia riparativa e mediazione penale, in Rassegna penitenziaria e criminologica, 1997.

Tramontano G. Percorsi di giustizia: verso una nuova modalità di risoluzione dei conflitti, in Rassegna penitenziaria e criminologica, 2010.

Viano E.C. Vittimologia oggi: i principali temi di ricerca e di politica pubblica, in Balloni E., Viano E.C., (a cura di), IV Congresso Mondiale di Vittimologia. Atti della giornata bolognese, Clueb, Bologna, 1989. 


\section{AUTHOR GUIDELINES}

Authors willing to publish original works on the "Rivista di Psicopatologia Forense, Medicina Legale, Criminologia", must submit them to the website http://pagepress.org/ socialsciences/index.php/psico/index; papers submitted to the journal will be evaluated by the Editorial Committee that can accept, refuse or accept them subject to reservation. Authors are requested to respect the following indications:

1. Full title of the paper;

2. name and surname of the author (s) on the first page; affiliations of each author.

3. "Riassunto" in Italian, "Abstract" in English and "Resumen" in Spanish, containing the reasons for the study, the main observations and conclusions of the author;

4. "Parole chiave" in Italian, "key words" in English and "palabras clave" in Spanish;

5. References shall be numbered consecutively in the order in which they are mentioned for the first time in the text, by numbers in brackets. References in the section "References" must be prepared as follows:

i) more than three authors, mention the first three authors plus "et al." If the document has only four authors, cite all authors;

ii) Title style: Use a capital letter only for the first word of the title.

Examples:

1. Waxman DA, Greenberg MD, Ridgely MS, et al. The effect of malpractice reform on emergency department care. New Engl J Med 2014;371:1518-25.

2. Buzzoni A. Responsabilità medica e sanitaria: nuovi profili penali e civili. FAD, Milano; 2014.

For Jurisprudential Review Articles, References are quoted in the text indicating the author's name followed by the year of publication (e.g., Granone, 1989; Waxman et al., 2014).

We also accept newsletters, useful news, original interviews, reviews, information on conferences and congresses.

By sending the original works, the Authors undertake not to publish the same work in another magazine, book or on the internet. For further information, please contact the Editorin-Chief at vincenzo.mastronardi@uniroma1.it.

\section{NORME DI PUBBLICAZIONE PER GLI AUTORI}

Chi vuole pubblicare lavori originali sulla "Rivista di Psicopatologia Forense, Medicina Legale, Criminologia", di cui l'accettazione avverrà previo esame di un Comitato Redazionale che può accettarli, rifiutarli o accettarli con riserva, deve sottometterli al sito http://pagepress.org/ socialsciences/index.php/psico/index, rispettando le seguenti indicazioni:

1. il titolo completo del lavoro;

2. nome e cognome dell'autore (o autori) in prima pagina; affiliazioni di afferenza di ogni autore.

3. Riassunto in italiano, "Abstract" in inglese e "Resumen" in spagnolo, contenenti le ragioni dello studio compiuto, le principali osservazioni e le conclusioni dell'Autore:

4. Parole chiave in italiano, "key words" in inglese e "palabras clave" in spagnolo;

5. Le Referenze devono essere numerate consecutivamente nell' ordine in cui sono citate per la prima volta nel testo mediante numeri posto fra parentesi. Nella sezione "Bibliografia" i riferimenti devono essere preparati come segue:

i) più di tre autori, indicare i primi 3 autori, et al. Se il documento ha solo 4 autori, citare tutti gli autori;

ii) stile del titolo: utilizzare una lettera maiuscola solo per la prima parola del titolo.

Esempi:

1. Waxman DA, Greenberg MD, Ridgely MS, et al. The effect of malpractice reform on emergency department care. New Engl J Med 2014;371:1518-25.

2. Buzzoni A. Responsabilità medica e sanitaria: nuovi profili penali e civili. FAD, Milano; 2014.

Per Articoli di Rassegna Giurisprudenziale, le Referenze vengono citate nel testo indicando il nome dell'autore seguito dall'anno di pubblicazione (ad es.: Granone, 1989; Waxman et al., 2014).

$\mathrm{Si}$ accettano anche notiziari, notizie utili, interviste originali, recensioni, informazioni su convegni e congressi.

Con l'atto dell'invio dei lavori originali, gli Autori si impegnano a non pubblicare lo stesso lavoro in altra rivista, libro $\mathrm{o}$ in internet. Per informazioni in proposito rivolgersi alla Direzione responsabile: vincenzo.mastronardi@uniroma1.it 

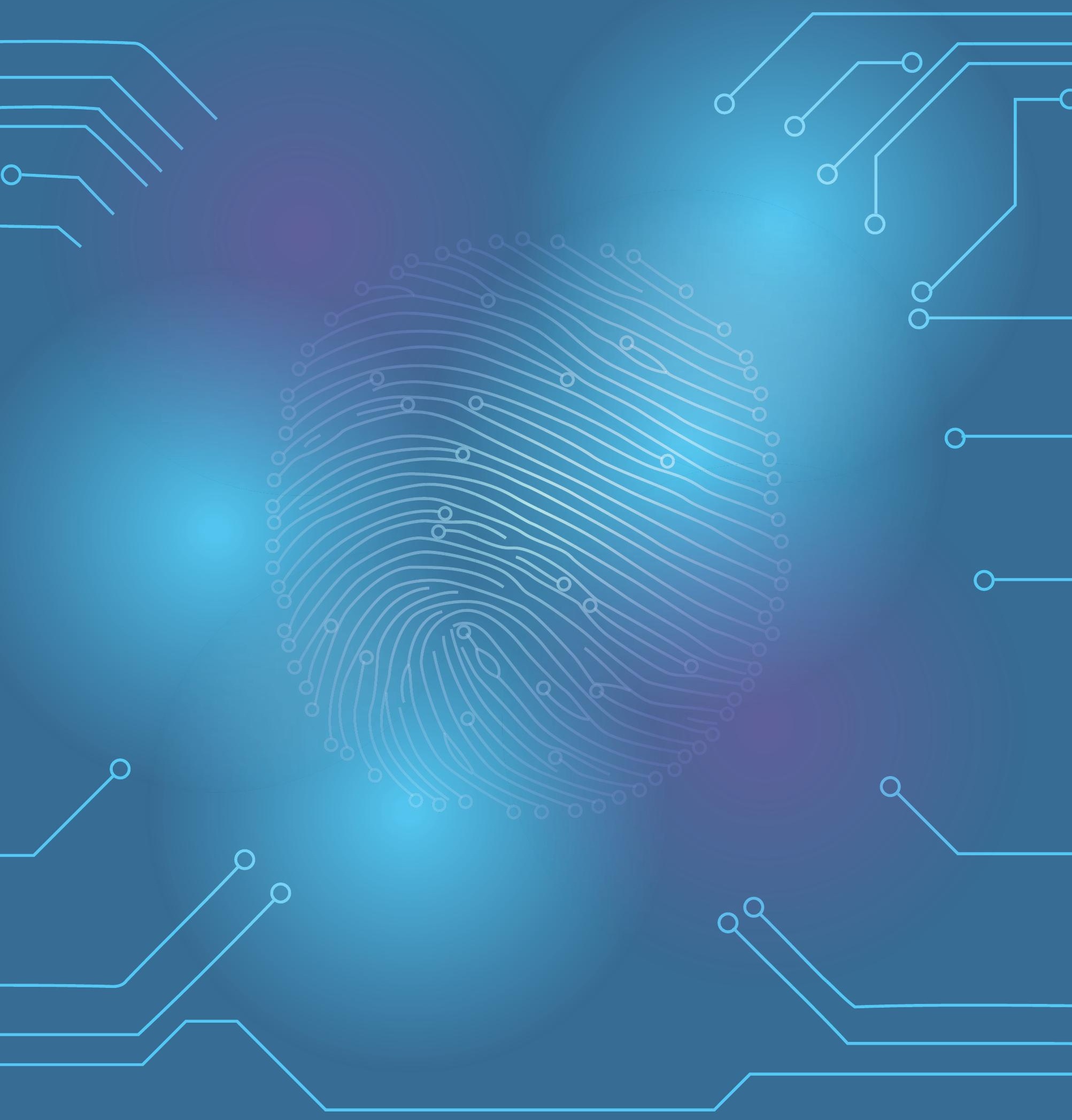

PAGEPress - Scientific Publications

Via A. Cavagna Sangiuliani, 5 - 27100 Pavia - Italy

Phone +390382 464340 - Fax +390382 34872

www.pagepress.org 